\title{
INFERENCES OF OCEAN CURRENTS FROM SEISMIC STREAMER POSITION DATA
}

\author{
TIMOTHY J. GRANT
}

\begin{abstract}
This paper describes the first attempt to infer ocean currents from the shapes of seismic streamers using real data. It demonstrates that it is feasible to infer the ocean currents, using a total least-squares solution at each shotpoint, when there is no lateral steering. There are some artifacts in the inferred currents when there is lateral steering, this is believed to be caused by errors in estimating the streamer velocity. The paper describes the residual equations that form the cost function and discusses how to choose weights in the cost function based on physical criteria. Correctly choosing weights is something of an art and requires further research to make automatic and robust.
\end{abstract}

Date: October 11, 2016.

Key words and phrases. Acquisition, Inverse Problem. 


\section{INTRODUCTION}

The ability to infer ocean currents along the length of seismic streamers may lead to several benefits for marine seismic surveys:

- Knowing the currents along the streamers in real time could lead to improvements in streamer steering.

- Improving the streamer steering could lead to efficiency and cost savings.

- Improving the streamer steering could allow the receiver positions to better match the receiver positions from previous surveys, and so improve reservoir monitoring (Kragh and Christie 2002).

- Inferring the currents could be incorporated into the receiver positioning algorithms to improve their accuracy.

Additionally, inferring the currents at a length scale that is shorter than the length of the streamers will provide valuable information on ocean currents to the oceanographic community. At present, currents on this length scale are poorly observed, being at a smaller scale than is presently observed using satellite altimetry; it is thought that processes on this smaller scale may play an important role in energy dissipation in the ocean and the mixing of gases and nutrients (Grant, Laws and Shuckburgh 2014).

The first paper to describe the possibility of inferring ocean currents from the shapes of streamers was by Polydorides, Storteig and Lionheart (2008); in this, and subsequent papers (Polydorides, Storteig and Lionheart 2009, and Polydorides and Storteig 2011) they assumed that there are tension measurements along the length of each streamer. Polydorides et al. (2008) show how (if there are no lateral steering devices on the streamers) the problem of inferring ocean currents can be reduced to differentiating noisy data. The theoretical work was extended by Polydorides et al. (2009) to include streamers with depth control units (DCUs) that provide lateral steering (as well maintaining the depths of the streamers). In this case it is not possible to reduce the problem to differentiating data, so a cost function is defined for the currents that is solved using a Newton method. Polydorides and Storteig (2011) returned to the problem of inverting for currents when there are no DCUs. They reformulated the problem of inferring the ocean currents in terms of Bayesian inference, so that, not only can the most likely solution for the currents be calculated, but given prior statistics concerning the currents, the probability density functions for the reconstructed currents can also be calculated. This enables confidence intervals to be placed on the reconstructed currents. They then discussed the problem of calculating the angle of attack that a streamer makes with the local currents along its length. They showed that the angle of attack is not Gaussian. Therefore, to estimate the angle of attack that the streamer makes with the local currents, they proposed using stochastic simulation. Given the means and covariances of the reconstructed currents, these distributions can be sampled to calculate the angle of attack and unbiased estimates of the mean and covariance can then be calculated. 
Grant et al. (2014) recognized that, in many commercial streamer systems, there are not tension measurements along the length of the streamers; instead there is typically a single measurement of the tension at the head of the streamer. The lack of tension measurements makes the inverse problem for the currents fundamentally underdetermined. To overcome this problem the authors exploited the fact that the vertical current velocities in the ocean are typically much less than the horizontal currents, so the currents can be considered to be horizontally divergence-free, therefore if an array of streamers is towed horizontally then it should still be theoretically possible to infer the currents.

1.1. Objective. The aim of this paper is to extend the work of Grant et al. (2014) by attempting to apply the idea to a real data set and, so, demonstrate that inferring currents is possible. This is the first attempt (to the author's knowledge) to infer the ocean currents from real streamer shape data.

Grant et al. (2014) assumed that the velocity of the streamer and its local angle were the observed variables. In truth the only available data are the tension at the head of each streamer, the currents recorded at the vessel by the acoustic Doppler current profiler (ADCP), the positions along the streamer, determined by an acoustic network at each shotpoint, and the time of each shotpoint. From these data, the velocities and angle must be calculated and used in the inverse model. How to do this in the presence of lateral steering is one of the challenges encountered in this paper.

As discussed, the currents are estimated by minimizing a cost function, and, so, to fulfill the main objective of inferring the currents, it is necessary to explore how the cost function should be weighted to obtain realistic currents. Unlike in previous numerical studies where the true currents are known, with real data we must develop criteria to judge whether the inferred currents are plausible or not.

The procedure, used in this paper, to infer the ocean currents is displayed in Figure 1.

\section{The MODEL FOR STREAMER DYNAMiCS}

To infer the currents from the shape of streamers, it is necessary to have a model of the streamer dynamics. The large-scale dynamics of a horizontally towed streamer can be modeled with the following two dimensional model (see Polydorides et al. $(2008,2009)$ and Grant et al. (2014) for a derivation of the model), which uses a local coordinate frame along the streamer, with axes in the tangential and normal directions to each point on the streamer. The independent variables are the time, $t$, and the arc length, $s$, (which is measured from the head to the tail of a streamer), and the dependent variables are

- $v_{t}$ and $v_{n}$ are the tangential and normal velocities of the streamer,

- $f_{t}$ is the tension in the streamer,

- $\theta$ is the angle the tangential direction, at a point on the streamer, makes with the $\mathrm{x}$ axis. 


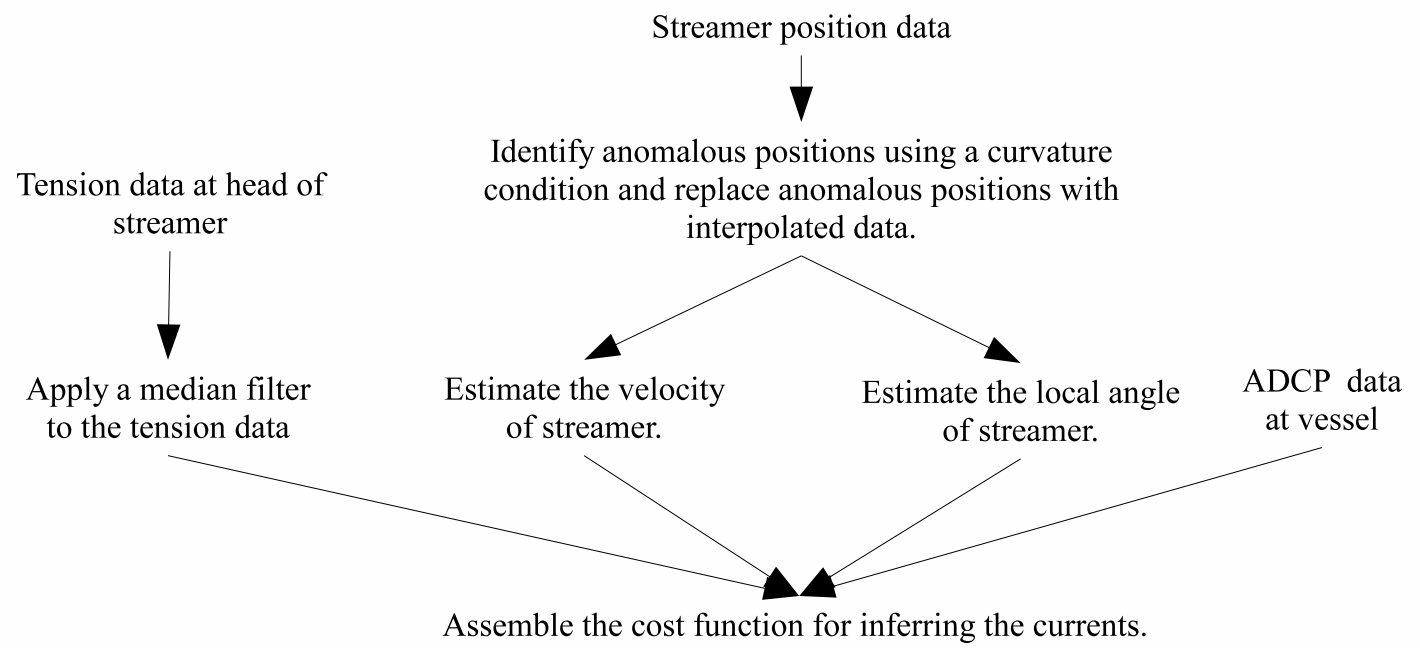

Fix all the weights, apart from the weights for the smoothness in space and horizontally divergence-free residuals.

Infer currents for a region of data, with no lateral steering, over a range of smoothness and divergence-free weights.

Pick the best smoothness and divergence-free weights by looking for coherent structures in time.

Infer currents for a region of data, when the lateral steering is suddenly turned on, over a range of weights for the velocity of the streamer and smoothness in time residuals.

Pick the best weights by eliminating jumps in the inferred currents when the lateral steering starts.

Infer the currents over the entire data set using the final choice of weights.

FiguRE 1. The procedure, used in this study, for inferring the currents.

The resulting model is

$$
\begin{aligned}
\frac{\partial v_{t}}{\partial s}-\frac{\partial \theta}{\partial s} v_{n} & =0, \\
\frac{\partial v_{n}}{\partial s}+\frac{\partial \theta}{\partial s} v_{t}-\frac{\partial \theta}{\partial t} & =0, \\
\frac{\partial f_{t}}{\partial s}-\frac{1}{2} \rho_{w} d \pi C_{t}\left(v_{t}-w_{t}\right)\left|v_{t}-w_{t}\right|+Q_{t}\left(s, t, v_{t}, v_{n} ; w_{t}, w_{n}\right) & =0, \\
\frac{\partial \theta}{\partial s} f_{t}-\frac{1}{2} \rho_{w} d C_{n}\left(v_{n}-w_{n}\right)\left|v_{n}-w_{n}\right|+Q_{n}\left(s, t, v_{t}, v_{n} ; w_{t}, w_{n}\right) & =0,
\end{aligned}
$$

where the first two equations are kinematic relations and the second two equations express the force balance in the tangential and normal directions respectively. The functions $Q_{t}$ and $Q_{n}$ express the forces provided by the DCUs (also referred to as birds). The parameters in the model are as follows:

- $d$ is the diameter of the streamer, 
- $\rho_{w}$ is the seawater density,

- $w_{t}$ and $w_{n}$ are the tangential and normal velocities of the currents,

- $C_{t}$ and $C_{n}$ are the tangential and normal drag coefficients.

For the streamer used in this test, $d=0.052 \mathrm{~m}$, and the sea water density (at the depth of the streamers) was measured at three points during the survey, yielding a best estimate of $\rho_{w}=1024.58 \mathrm{~kg} \cdot \mathrm{m}^{-3}$. The boundary conditions for the model are that the velocity of the tow point is known, that the normal velocity of the tail point is that of the normal current at the tail, and that the tail buoy is assumed to provide a constant drag force, denoted by $T_{L}$, if its speed through the water is $2.57 \mathrm{~m} / \mathrm{s}$ ( 5 knots). A mechanic on board the vessel that conducted the survey suggested that $T_{L}=1500 \mathrm{~N}$ is the best estimate of the tail buoy drag at 5 knots. The tail buoy drag is expressed as

$$
\frac{T_{L} \sqrt{\left(v_{t}-w_{t}\right)^{2}+\left(v_{n}-w_{n}\right)^{2}}\left|v_{t}-w_{t}\right|}{2.5722^{2}} .
$$

In the field test, each of streamers 1 to 4 and 8 to 12 had a front float between the first receiver position and the tow adapter (where the tension is the streamer is recorded) that contributes to the recorded tension. The drag caused by the front float is modeled in exactly the same manner as the tail buoy. Additionally, these same streamers had miniwings (small deflectors designed to pull the streamers out of the wake caused by the large deflector) between the tow adapters and first receiver positions; these are assumed to provide a constant drag force, denoted by $M$, at $2.57 \mathrm{~m} / \mathrm{s}$ (5 knots), and so are modeled using

$$
\frac{M \sqrt{\left(v_{t}-w_{t}\right)^{2}+\left(v_{n}-w_{n}\right)^{2}}\left|v_{t}-w_{t}\right|}{2.5722^{2}} .
$$

The amount of drag produced by the miniwings depends on how they are set up for a given survey; for this survey the most appropriate value for the miniwing drag is $M=450 \mathrm{~N}$.

2.0.1. The drag coefficients. The model for the streamer dynamics depends on the tangential and normal drag coefficients, which are not known. In order to calculate the drag coefficients, the speed of the streamer through the water needs to be known, this was accomplished by using a crude estimate of the ocean currents based on the ADCP data, and using the position and time data to calculate the velocity of the streamer. The tangential drag coefficient was then obtained by fitting the estimated drag to the recorded tension; the normal drag coefficient was estimated by exploiting a vessel turn, that produced significant curvature in the streamer that was fitted to the normal hydrodynamic force. The resulting estimates are shown in Table 1. There is a symmetric pattern in the drag coefficients, about the centre of the streamer spread. This pattern in the drag on the streamers is believed to be caused by differences in barnacle growth on the streamers. Therefore it seems appropriate to treat the streamers as having different drag coefficients. To be accurate, the drag coefficients should be treated as varying along the length of each streamer because the number of barnacles will change on different sections of each streamer due to their location and the different times at which sections are cleaned. In this study, the drag coefficients will be treated as a single constant along each streamer. This is due to the fact that there is a single tension measurement per streamer, so, it is not clear how to 


\begin{tabular}{|l||c|c|c|c|c|c|c|c|c|c|c|c|}
\hline Streamer & 1 & 2 & 3 & 4 & 5 & 6 & 7 & 8 & 9 & 10 & 11 & 12 \\
\hline \hline$C_{t}$ & 0.0036 & 0.0042 & 0.0040 & 0.0034 & 0.0037 & 0.0037 & 0.0037 & 0.0037 & 0.0032 & 0.0039 & 0.0039 & 0.0033 \\
$C_{n}$ & 1.3447 & 1.6017 & 1.5966 & 1.3645 & 1.3425 & 1.2821 & 1.3458 & 1.3397 & 1.2361 & 1.4178 & 1.4367 & 1.2522 \\
\hline
\end{tabular}

TABLE 1. The best estimates of the drag coefficients assuming $T_{L}=1500 \mathrm{~N}$ and $M=450 \mathrm{~N}$.

constrain the varying values of the drag coefficients along the streamers if they are allowed to be functions of arc length. If, instead of solving for the currents at each individual shotpoint, but, rather, over a long timewindow consisting of many shotpoints (so this would not be a real time process to aid steering), then by treating the drag coefficients as functions of arc length only, and not time, it may be possible to estimate them. Instead of a single unknown which we find at every shotpoint (constrained to be close to our prior estimate) we would gain a number of unknowns (depending on the spatial sampling of the streamer). This at first seems to make the problem more underdetermined, however, these unknowns must be the same at every time step. So, provided we have more time steps than additional unknowns, we will actually constrain the model better, leading to better current estimates as well as knowing how the drag coefficients vary along the streamer. This could then be used as a diagnostic as to determine which sections of streamers must be cleaned next. ${ }^{1}$

2.1. Key assumptions about the ocean currents. The ocean currents are inferred along the streamers by discretizing equations (3) and (4) and fitting the currents and data to them in a cost function. Grant et al. (2014) showed how the problem of determining the ocean currents is under-determined due to a lack of tension measurements along the streamer, and so, additional assumptions about the currents must be made. These are

- The currents should be smooth across and along the streamer array. This assumption is largely to remove the effects of having noisy position and tension data.

- Structures in the ocean currents should be changing slowly relative to the speed of the towing vessel, which is traveling at approximately $2.57 \mathrm{~m} . \mathrm{s}^{-1}$ (5 knots). This will primarily be used as a criteria to judge whether the inferred currents are plausible or not.

- The currents should be close to the average value recorded by the ADCP on the vessel over the previous hour. The vessel takes about an hour to travel the length of a streamer; therefore, under the assumption that the ocean currents change slowly in time, the previous hour's ADCP record gives a good prior estimate as to what the currents should be along the streamers. The primary result of this assumption is to cause there to be a reasonable tension estimate along each streamer. Given a reasonable tension estimate along each streamer, the curvature of each streamer provides the information needed to infer the currents flowing across each streamer.

\footnotetext{
${ }^{1}$ Suggested by Robert Laws (Schlumberger Gould Research)
} 
- Finally, it is assumed that the currents should be approximately horizontally divergencefree because the currents in the ocean are primarily two dimensional; typically, the velocities of the horizontal currents are at least a couple of orders of magnitude greater than the velocities of the vertical currents (Thomas, Tandon and Mahadevan 2008; Vallis 2006; Grant et al. 2014). This assumption allows us to infer the in-line currents.

\section{DAtA PROCESSING}

The data for this work were acquired by the Western Neptune in February 2013, off the coast of Brazil (near the Iara and Juptier hydrocarbon reserves ${ }^{2}$ ), and consists of

- tension measurements from the head of each streamer at each shotpoint (roughly every $10 \mathrm{~s})$

- calculated receiver positions (12.5 m apart),

- the DCU black box data that describes the roll angle and the port and starboard control angles of each DCU at each shotpoint,

- ADCP measurements of the currents at the vessel location, every $30 \mathrm{~s}$,

- three conductivity temperature and depth oceanographic probe dips were undertaken during the study from which the sea water density, at the depth of the streamers, was estimated.

3.1. Anomalous tension data. Figure 2 shows the raw tension measurements (in blue) for Streamer 11. There are several instances when the variations in the raw tension are far greater than normal; this is most obvious after shot 2000 (which, if a true measurement, would suggest that there is only a very small difference between the in-line velocity of the streamer and the ocean currents: this is very unlikely). A median filter, with a 25 -shot window, was used (on the tension data from all the streamers) to remove these artifacts without over-smoothing the data. The red line in Figure 2 shows the result of applying this filter to the tension record. The filtered tension data are used as the input, in the cost function to infer the currents.

3.2. Problems with calculated receiver positions. Most of the calculated receiver positions are interpolated from the positions of the hydrophones included in an acoustic network. If the calculated receiver positions are used to estimate the curvature, then, artifacts appear in the curvature that move with the streamer; these are unlikely to be due to currents but rather the effects of the interpolation scheme and further processing. This unwanted signal in the streamer's curvature will mask small-scale features in the currents. To alleviate this, only the calculated receiver positions that were closest to members of the acoustic network (receivers and transmitters) were used to infer the currents, this is because these are assumed to be the most accurate locations; the other calculated positions were discarded. This increases the spatial separation of position data, on a streamer, from $12.5 \mathrm{~m}$ to around $70 \mathrm{~m}$.

The tail buoy position is required to infer the currents because it provides the point to impose the empirical tail buoy relation. However, when calculating the angle along a streamer, there can be a noticeable jump in the angle at the tail so as to accommodate the tail buoy position.

\footnotetext{
${ }^{2}$ No seismic data were acquired during this experiment.
} 


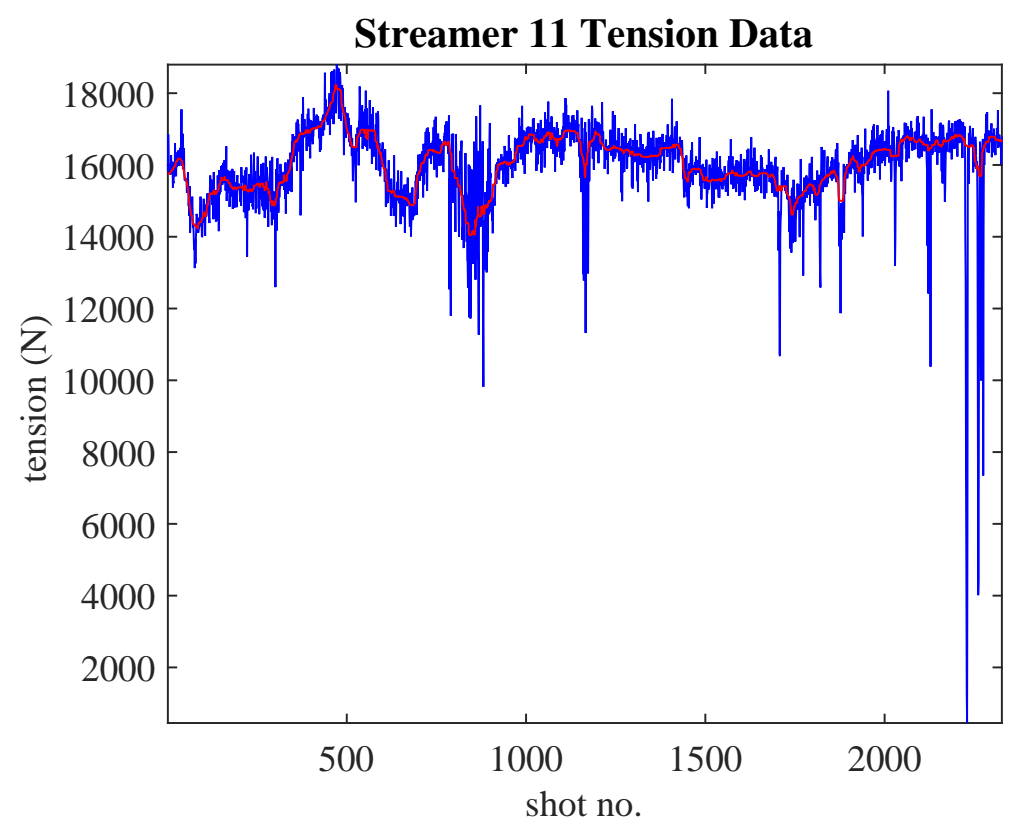

Figure 2. The raw tension measurements on Streamer 11 (blue) and the processed tension using a median filter (red) with a 25 -shot window

This suggests that tail buoy position data has be processed differently to the other positions or that the difference is because the tail buoy is at the sea surface whereas the streamers are being towed at a depth of $9 \mathrm{~m}$. In either case, the tail buoy positions are not reliable for the purposes of inferring the currents at the depth of the streamers. So, when inferring the currents, the tail buoy locations will be treated with a greater degree of uncertainty than the other positions.

Vessel turns and cross currents produce curvature in the streamers. It is the curvature that provides the signal required to infer the cross currents. The curvature must be calculated from the position data; errors in the position data will, therefore, lead to errors in the inferred currents. Therefore, in the inverse scheme the positions are treated as having errors. It is important to note that these errors are not independent; they are correlated along and across the streamer array. Therefore, to ensure that these errors are treated correctly, the acoustic network equations are mimicked in the cost function.

3.2.1. Discrete notation. Having restricted our attention to only the position data that relates to the acoustic network, let us use the following labeling system. Let us refer to the calculated receiver positions that are being used to mimic the acoustic network as nodes.

- Let $f_{t j, k}^{i}, x_{j, k}^{i}, y_{j, k}^{i}$ refer to the tension, $x$ and $y$ coordinates respectively, of the $k^{\text {th }}$ node (labeling from the head to the tail of the streamer), on the $j^{\text {th }}$ streamer (labeling from starboard to port), at the $i^{\text {th }}$ shotpoint.

- Let $w_{x j, k}^{i}, w_{y_{j, k}}^{i}, v_{x j, k}^{i}$ and $v_{y_{j, k}}^{i}$ be the $x$ and $y$ components of the currents and the streamer, respectively, at each node. We wish to infer the currents, and the velocity of the cable must be calculated from the position data at different shotpoints.

- Let there be $K$ positions on each streamer.

- Let $t^{i}$ denote the time of the $i^{\text {th }}$ shotpoint. 
Having labeled the data the following differences are defined:

$$
\begin{aligned}
& \Delta t^{i}=t^{i+1}-t^{i}, \\
& \Delta x_{j, k}^{i}=x_{j, k+1}^{i}-x_{j, k}^{i}, \quad \Delta y_{j, k}^{i}=y_{j, k+1}^{i}-y_{j, k}^{i}, \quad \Delta s_{j, k}^{i}=\sqrt{\Delta^{\Delta x_{j, k}^{i}{ }^{2}+\Delta y_{j, k}^{i}{ }^{2}},} \\
& \delta x_{j, k}^{i}=x_{j+1, k}^{i}-x_{j, k}^{i}, \quad \delta y_{j, k}^{i}=y_{j+1, k}^{i}-y_{j, k}^{i}, \quad \delta c_{j, k}^{i}=\sqrt{\delta x_{j, k}^{i}{ }^{2}+\delta y_{j, k}^{i}{ }^{2}} .
\end{aligned}
$$

The distance of a node along the streamer (arc length) is then given by

$$
s_{j, k}^{i}=\sum_{l=1}^{k-1} \Delta s_{j, k}^{i},
$$

and the local angle of the streamer (for $\Delta x_{j, k}^{i}>0$ ) is given by

$$
\theta_{m j, k}^{i}=\tan ^{-1}\left(\Delta y_{j, k}^{i} / \Delta x_{j, k}^{i}\right)
$$

This is most naturally interpreted as the angle at the midpoint between two nodes. The angle at the nodes is obtained through averaging at the internal points

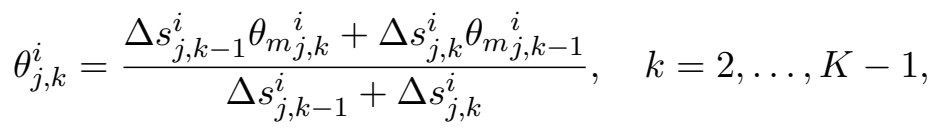

and projecting at the end points (because the data have been processed, jumps in the angle at the DCUs may have been smoothed out; therefore, the angle at the node either side of a DCU is calculated by projecting in the same manner as at the end nodes),

$$
\begin{aligned}
\theta_{j, 1}^{i} & =2 \theta_{m j, 1}^{i}-\theta_{j, 2}^{i}, \\
\theta_{j, K}^{i} & =2 \theta_{m j, K-1}^{i}-\theta_{j, K-1}^{i} .
\end{aligned}
$$

Having calculated the local angle along each streamer, the tangential and normal velocities of the currents and the streamer are given by

$$
\begin{gathered}
w_{t j, k}^{i}=w_{x j, k}^{i} \cos \theta_{j, k}^{i}+w_{y_{j, k}}^{i} \sin \theta_{j, k}^{i}, \quad w_{n j, k}^{i}=-w_{x j, k}^{i} \sin \theta_{j, k}^{i}+w_{y_{j, k}}^{i} \cos \theta_{j, k}^{i}, \\
v_{t j, k}^{i}=v_{x j, k}^{i} \cos \theta_{j, k}^{i}+v_{y_{j, k}^{i}}^{i} \sin \theta_{j, k}^{i}, \quad v_{n j, k}^{i}=-v_{x j, k}^{i} \sin \theta_{j, k}^{i}+v_{y_{j, k}^{i}}^{i} \cos \theta_{j, k}^{i},
\end{gathered}
$$

respectively.

3.2.2. Identifying anomalous points through curvature. Having restricted the position data to calculated receiver positions close to acoustic network locations, there remains anomalous data. These are apparent as kinks in the streamer shapes that do not correspond to the position of a DCU providing significant lateral steering. These questionable points can be automatically identified as follows (treating each streamer and time step separately):

(1) Calculate the curvature along the the streamer at a given time step by raw differencing,

$$
\kappa_{j, k}^{i}=\frac{\theta_{m_{j, k+1}^{i}}^{i}-\theta_{m_{j, k}^{i}}^{i}}{\frac{1}{2}\left(\Delta s_{j, k+1}^{i}+\Delta s_{j, k}^{i}\right)}, \quad k=1, \ldots, K-2 .
$$



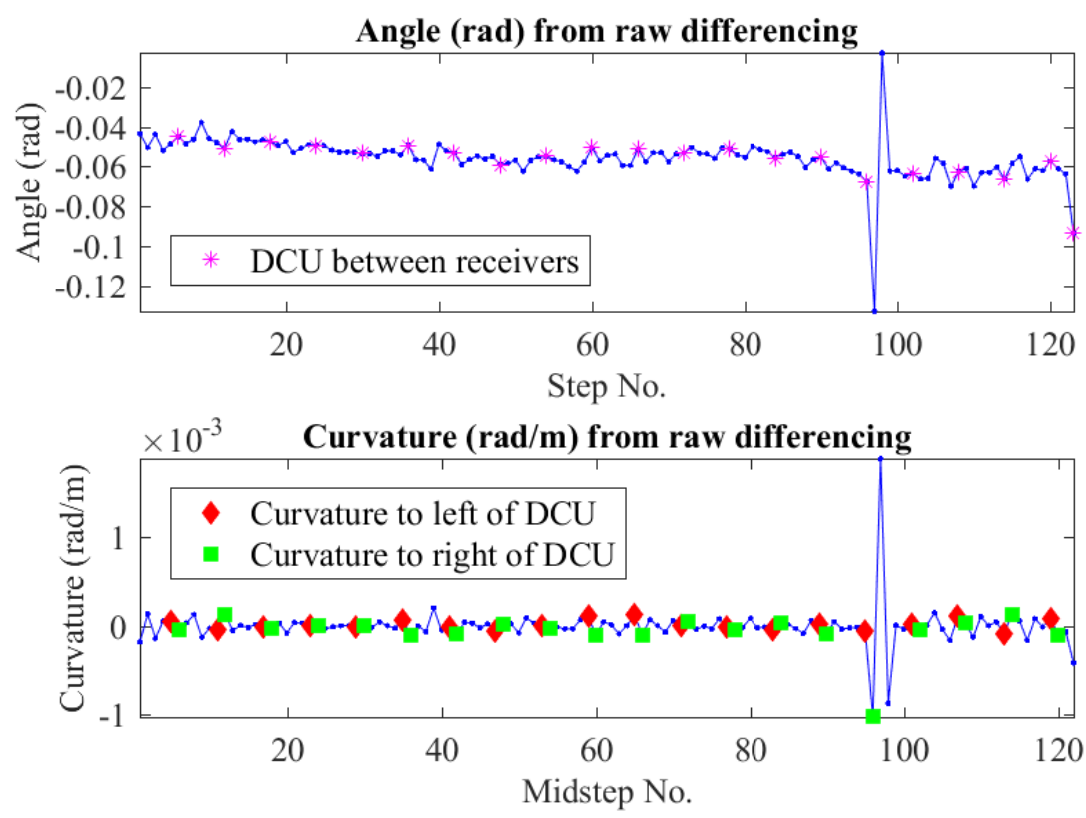

Figure 3. An example of identifying an anomalous position by using the curvature. Receiver 98 appears to be out of position (the jump in curvature does not correspond to a DCU position providing a significant lateral force). This leads to spuriously high normal currents being inferred near this point, unless this receiver position is replaced by interpolated data when inferring the currents.

(2) Calculate the mean and standard deviation of the curvature excluding positions associated with the DCUs (we can expect to see high curvature here if there is significant lateral steering) and excluding the tail buoy position (based on the observation descried in $\S 3.2)$.

(3) Identify points (excluding those associated with the DCUs) that have a curvature that deviates from the mean by some threshold; for this work four standard deviations of the curvature were chosen (see Figure 3 for an example of such a point).

(4) Replace the positions of these points by interpolating the position data using the arc length, $s$, as the independent variable. In this study I used cubic spline interpolation.

3.3. Estimating the velocity. Calculating the velocity of a streamer from the position data is the problem of differentiating noisy data. Following Hanke-Bourgeois and Scherzer (2001) and Chartrand (2011) a cost function method is used to find the velocity of each individual node. The cost of fitting the time integral of the velocity to the position data is minimized subject to the constraint that the acceleration must be small.

Let $\mathbf{x}_{j, k}$ and $\mathbf{y}_{j, k}$ denote column vectors of the $x$ and $y$ locations of the $k^{\text {th }}$ node on the $j^{\text {th }}$ streamer over all the time steps. The discrete integral operator and difference operator are defined below. The discrete integral operator here uses the trapezoidal rule, so, the calculated velocities coincide with the same times as the position data; if the midpoint rule was used instead, then the calculated velocities would naturally be at the mid-time points and the 
difference operator would require adjusting.

$$
\begin{gathered}
\mathbf{A}=\left(\begin{array}{ccccc}
0 & 0 & 0 & 0 & \ldots \\
\frac{\Delta t^{1}}{2} & \frac{\Delta t^{1}}{2} & 0 & 0 & \ldots \\
\frac{\Delta t^{1}}{2} & \frac{\Delta t^{1}+\Delta t^{2}}{2} & \frac{\Delta t^{2}}{2} & 0 & \ldots \\
\frac{\Delta t^{1}}{2} & \frac{\Delta t^{1}+\Delta t^{2}}{2} & \frac{\Delta t^{2}+\Delta t^{3}}{2} & \frac{\Delta t^{3}}{2} & \ldots \\
\vdots & \vdots & \vdots & \vdots & \ddots
\end{array}\right), \\
\mathbf{D}=\left(\begin{array}{cccccc}
-\frac{1}{\Delta t^{1}} & \frac{1}{\Delta t^{1}} & 0 & 0 & 0 & \ldots \\
0 & -\frac{1}{\Delta t^{2}} & \frac{1}{\Delta t^{2}} & 0 & 0 & \ldots \\
0 & 0 & -\frac{1}{\Delta t^{3}} & -\frac{1}{\Delta t^{3}} & 0 & \ldots \\
0 & 0 & 0 & -\frac{1}{\Delta t^{4}} & \frac{1}{\Delta t^{4}} & \ldots \\
\vdots & \vdots & \vdots & \vdots & \vdots & \ddots
\end{array}\right) .
\end{gathered}
$$

Letting $\hat{x}_{j, k}^{i}=x_{j, k}^{i}-x_{j, k}^{1}$ and $\hat{y}_{j, k}^{i}=y_{j, k}^{i}-y_{j, k}^{1}$, the cost function to calculate the velocities is

$$
J\left(\mathbf{v}_{\mathbf{y}_{j, k}}\right)=\left(\mathbf{A} \mathbf{v}_{\mathbf{y}_{j, k}}-\hat{\mathbf{y}}_{j, k}\right)^{T}\left(\mathbf{A v}_{\mathbf{y}_{j, k}}-\hat{\mathbf{y}}_{j, k}\right)+w\left(\mathbf{D v}_{\mathbf{y}_{j, k}}\right)^{T}\left(\mathbf{D v}_{\mathbf{y}_{j, k}}\right),
$$

where $w$ is a weight we are free to choose. Thus, the velocities are

$$
\mathbf{v}_{\mathbf{x} j, k}=\left(\mathbf{A}^{T} \mathbf{A}+w \mathbf{D}^{T} \mathbf{D}\right)^{-1} \mathbf{A}^{T} \hat{\mathbf{x}}_{j, k}, \quad \mathbf{v}_{\mathbf{y}_{j, k}}=\left(\mathbf{A}^{T} \mathbf{A}+w \mathbf{D}^{T} \mathbf{D}\right)^{-1} \mathbf{A}^{T} \hat{\mathbf{y}}_{j, k} .
$$

How the weight is chosen is somewhat subjective. A sensible criteria is to increase the weight (for a given node) until some signal is apparent in the calculated acceleration, as can be seen in Figure 4. Increasing the weight beyond this may cause signal to be lost in the velocity data. This is easiest to see in the right hand column of Figure 4, note that the $y$ acceleration, in the bottom panel, is noisy but we can discern a signal that corresponds to the smoothed velocity, in the top panel. For smaller weights the acceleration signal cannot be distinguished from noise, and for greater weights the acceleration data looks smoother but the amplitudes of the smoothed velocity data is reduced near the minimum and maximum values. Having chosen the weight, the procedure is repeated for all the other nodes. Despite treating each node separately, the resulting velocities appear smooth in space (Figure 5). To speed up the process of calculating the velocities, the data were windowed and the overlapping portions were experientially chosen so that jumps due to the windowing did not occur in the acceleration.

By applying the discrete integral operator to the velocities, positions that are consistent with the smoothed velocities are obtained (i.e., the positions that, by differencing in time, would produce the smooth velocities). Whilst these smoothed positions are not used to infer the currents, they are, nevertheless useful. Examining the changes in position,

$$
\mathbf{A}^{T} \mathbf{v}_{\mathbf{x} j, k}-\hat{\mathbf{x}}_{j, k}, \quad \mathbf{A}^{T} \mathbf{v}_{\mathbf{y}_{j, k}}-\hat{\mathbf{y}}_{j, k},
$$

can indicate where signal in the velocity may have been lost. Figures 6 and 7 show these differences. Clearly, the magnitude of the changes is generally greatest near the centre of the streamer spread. This is consistent with the positioning errors from the acoustic network being greatest near the centre of the array. However, Figure 7 shows an example when this is not the case; it indicates that when sudden lateral steering is applied the expected jump in velocity of 

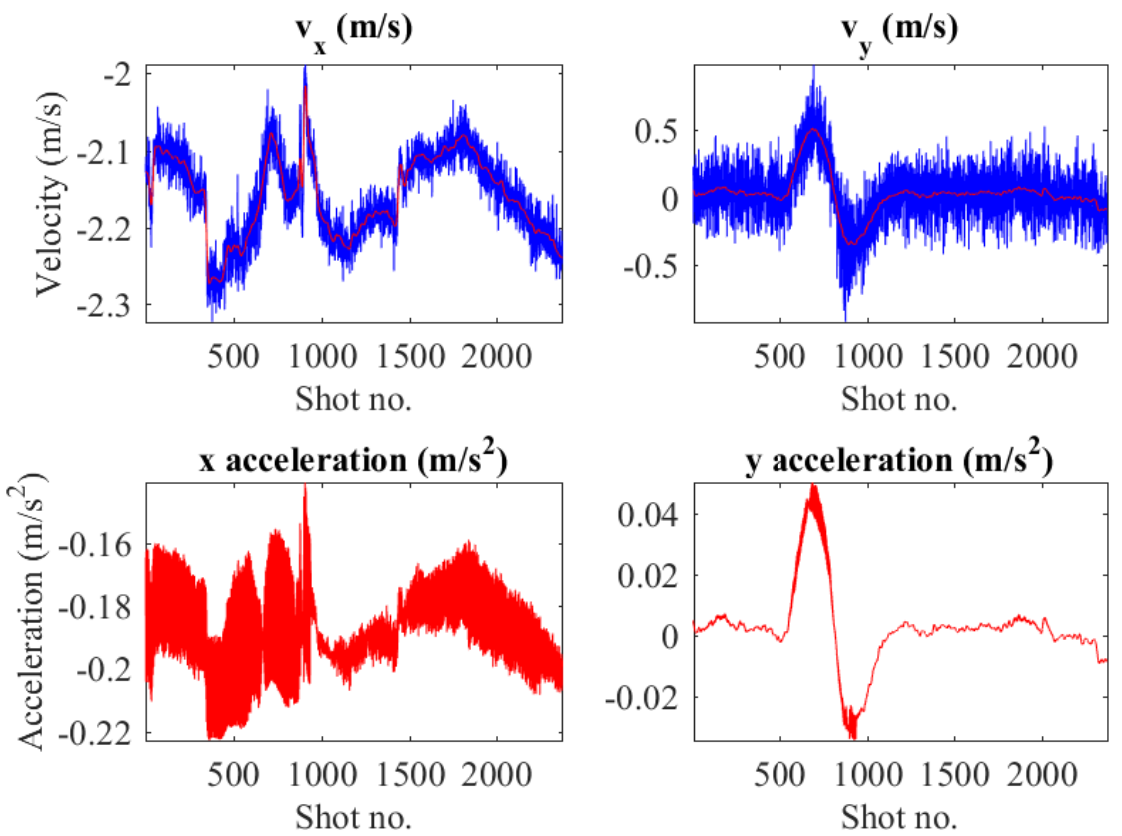

Figure 4. The calculated velocity of a node from raw differencing (blue) and the cost function method (red), where the weight was chosen so that a signal was apparent in the estimated acceleration.

the streamer is smoothed out. This error may affect the processed streamer velocities before and after the lateral steering is applied. Because it is apparent that the processed velocities contain errors, when inverting for the ocean currents, the velocities should be treated as unknowns which are constrained to be close to the processed values. 


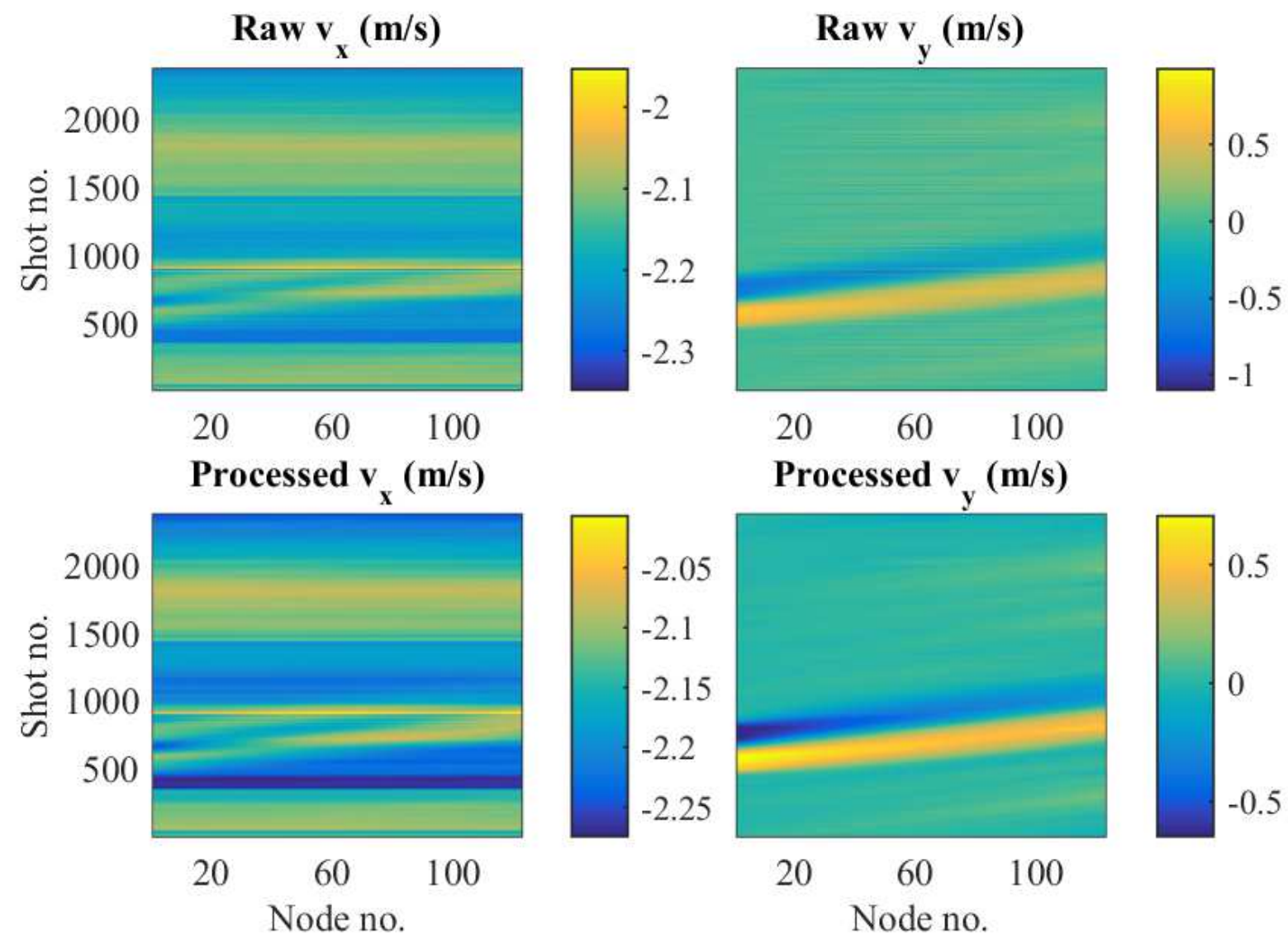

Figure 5. The calculated velocities $(\mathrm{m} / \mathrm{s})$ along a streamer by raw differencing (top row) and the cost function method (bottom row). Note the change in the colour scale between the raw and the processed velocities. 

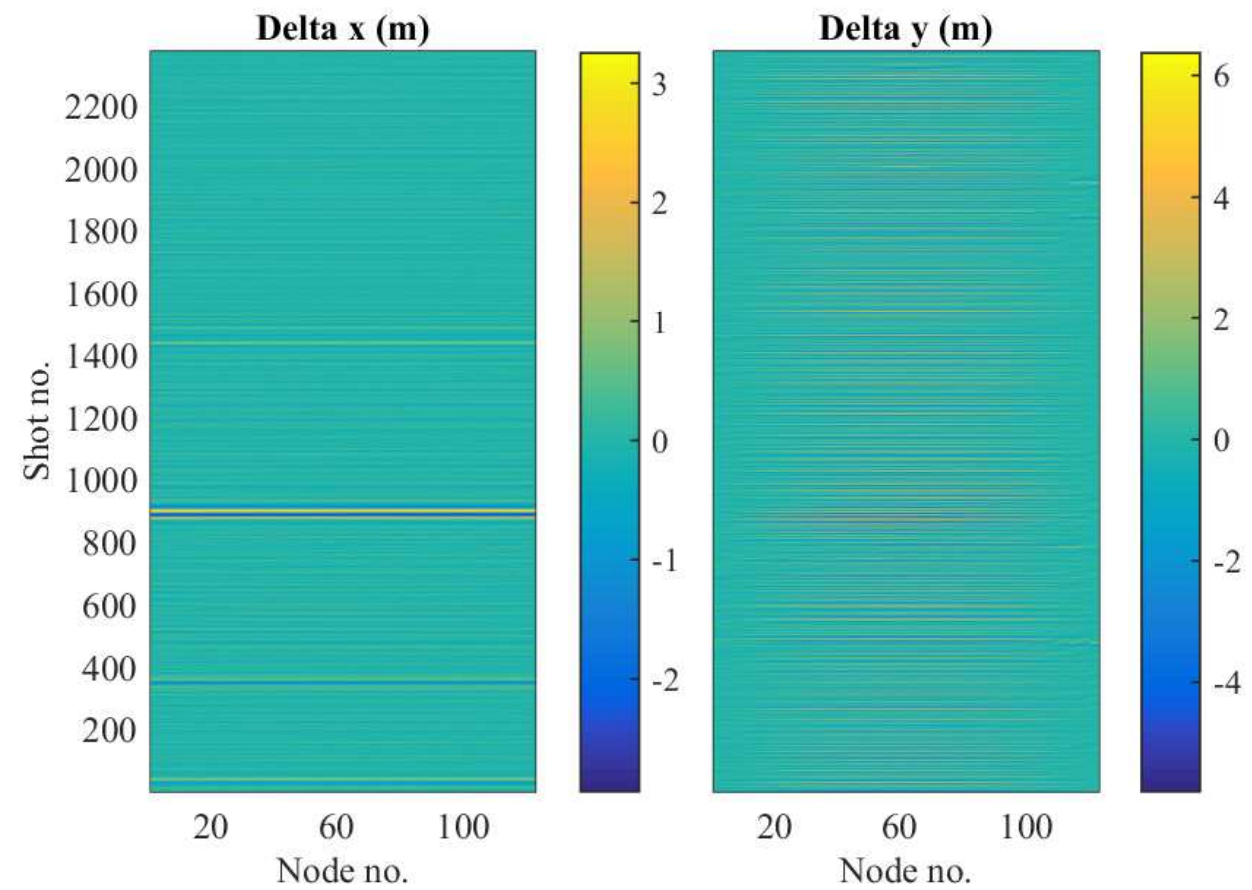

FiguRE 6 . The change in position $(\mathrm{m})$ required to obtain the smooth velocities for streamer 6 . Note that the largest changes generally occur near the centre of the streamer, this is where there is the greatest uncertainty in the position data. 

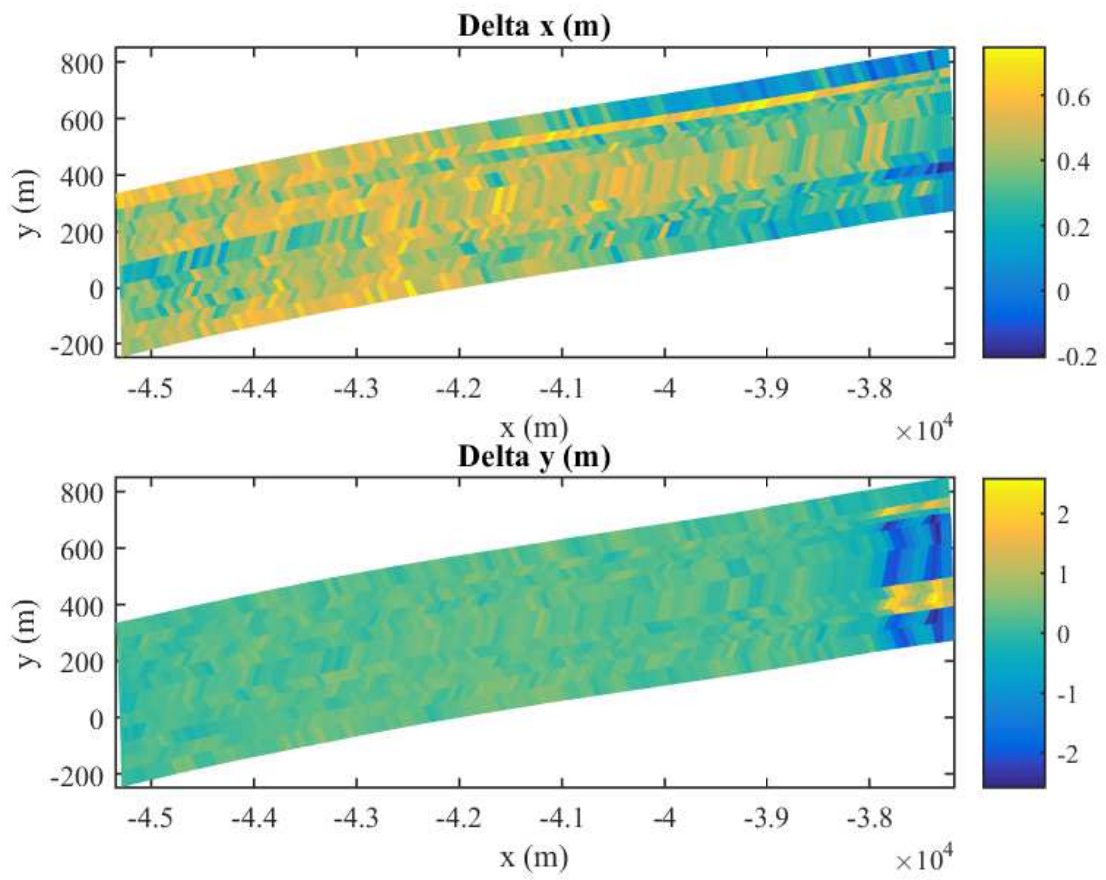

Figure 7 . The change in position (m), at a single shotpoint, required to obtain the smooth velocities. Note that, at this shot the maximum changes are at the tail of the streamers rather than at the centre of the streamers (where there is the greatest position uncertainty). At this shot the rear DCUs suddenly provided a large amount of lateral steering on Streamers 2 - 12. The change in position near the tail suggests that the expected jump in velocity due to this steering may have been lost in the processing. 


\section{INVERTING FOR CURRENTS}

The currents are inferred using a total least-squares solution (Wunsch, 2006) at each shotpoint. The residual equations for the cost function incorporate our knowledge of the system and beliefs about the errors in the data we have.

The curvature and velocity of the streamer are caused by the vessel's motion and the ocean currents. Therefore the currents are found by fitting them to the model of the streamer dynamics, given by equations (1) to (4). However, the velocity and position data for the streamers contain errors, so, in the cost function, the velocity and position are treated as unknowns, and residual equations are included that constrain these variables to be close to the best estimates we have for them. Also, the model for the streamer dynamics depends on parameters of which we are uncertain, such as the drag coefficients, so, these too are treated as unknowns that extra residual equations force to be close to their prior values, and additional equations enforce any relations between them. Finally, as the problem is under-determined, to regularize it, discrete versions of the assumptions about the ocean currents (\$2.1) are included as residual equations in the cost function. Section 4.1 describes the discrete equations that form the residual equations and how they are combined and weighted in the cost function.

4.1. Assembling the cost function. The discrete residual equations, that form the cost function, are presented below. The Jacobian for the residual equations is constructed by repeated applications of the chain rule.

\subsubsection{The single streamer residuals.}

- The main equations.

\section{The DCU forces}

To infer the currents, we must be able to calculate the forces provided by the DCUs, for a given set of control angles and currents. The DCUs used in this field test consist of two fins, lying in the same plane, which are able to rotate around the streamer. There are three control angles that are recorded at each shotpoint: $\psi_{\text {roll }}$, the roll angle (if the roll angle is 0 rad then the fins are horizontal and provide only a vertical force; if the roll angle is $\pm \pi / 2 \mathrm{rad}$ then the fins are vertical and provide only a lateral force), $\psi_{\text {port }}$ and $\psi_{s t b d}$, which are control angles that determine the angle of attack for the port and starboard fins. The force per unit length that each DCU produces depends on the diameter of the fins $\phi$ and three confidential parameters, $C_{\text {lift }}, C_{d r a g}$ and $C_{c o n}$, which have been determined in laboratory experiments.

Adapting Polydorides et al. (2009), who assumed that the DCU fins were vertical or horizontal only, the fin forces are modelled as follows (assuming that there are no vertical currents). If the roll angle is zero, the fins are horizontal and the flow across the fin is purely due to the tangential currents. If however the fins are vertical the (horizontal) normal currents contribute to the flow across the fins. The component of the flow, in the direction perpendicular to the plane the DCU's fins are lying in, is

$$
v_{\text {perp }}=\left(v_{n}-w_{n}\right) \sin \left(\psi_{\text {roll }}\right) .
$$


The flow makes an angle with the DCU fins

$$
\psi_{\text {flow }}=\tan ^{-1}\left(\frac{v_{\text {perp }}}{v_{t}-w_{t}}\right),
$$

hence the angle of attack, for each fin, is the sum of the angle the flow makes with the fins and the control angle:

$$
\alpha_{\text {port }}=\psi_{\text {port }}+\psi_{\text {flow }}, \quad \alpha_{\text {stbd }}=\psi_{\text {stbd }}+\psi_{\text {flow }} .
$$

The lift and drag force are then calculated using the angles of attack and flow speed

$$
\begin{aligned}
Q_{L} & =\frac{\rho_{w}}{2} \phi C_{\text {lift }}\left(\alpha_{\text {port }}+\alpha_{\text {stbd }}\right)\left(v_{\text {perp }}^{2}+\left(v_{t}-w_{t}\right)^{2}\right), \\
Q_{D} & =\frac{\rho_{w}}{2} \phi\left(C_{\text {drag }}\left(\alpha_{\text {port }}^{2}+\alpha_{\text {stbd }}^{2}\right)+2 C_{\text {con }}\right)\left(v_{\text {perp }}^{2}+\left(v_{t}-w_{t}\right)^{2}\right) .
\end{aligned}
$$

The lift and drag are in the normal and tangential directions to the flow respectively (Lighthill 1986). So the fin forces are

$$
\begin{aligned}
q_{t} & =Q_{D} \cos \left(\psi_{\text {flow }}\right)-Q_{L} \sin \left(\psi_{\text {flow }}\right), \\
q_{n} & =\sin \left(\psi_{\text {roll }}\right)\left(Q_{D} \sin \left(\psi_{\text {flow }}\right)+Q_{L} \cos \left(\psi_{\text {flow }}\right)\right), \\
q_{b} & =\cos \left(\psi_{\text {roll }}\right)\left(Q_{D} \sin \left(\psi_{\text {flow }}\right)+Q_{L} \cos \left(\psi_{\text {flow }}\right)\right),
\end{aligned}
$$

where $q_{t}$ and $q_{n}$ are, respectively, the tangential and normal forces, and $q_{b}$ is the force in the binormal (vertical) direction which is not used in this study.

The force equations depend on the relative velocity between the streamers and the ocean currents between the nodes. These are given by

$$
\begin{aligned}
& v_{r t_{j, k+1 / 2}}^{i}=\left(\frac{v_{x_{j, k}^{i}}^{i}+v_{x j, k+1}^{i}}{2}-\frac{w_{x_{j, k}}^{i}+w_{x j, k+1}^{i}}{2}\right) \cos \left(\theta_{m_{j, k}}^{i}\right)+ \\
& +\left(\frac{v_{y_{j, k}^{i}}^{i}+v_{y_{j, k+1}^{i}}^{i}}{2}-\frac{w_{y_{j, k}^{i}}^{i}+w_{y_{j, k+1}^{i}}^{i}}{2}\right) \sin \left(\theta_{m_{j, k}}^{i}\right), \\
& v_{r n j, k+1 / 2}^{i}=-\left(\frac{v_{x j, k}^{i}+v_{x j, k+1}^{i}}{2}-\frac{w_{x j, k}^{i}+w_{x j, k+1}^{i}}{2}\right) \sin \left(\theta_{m j, k}^{i}\right)+ \\
& +\left(\frac{v_{y_{j, k}}^{i}+v_{y_{j, k+1}}^{i}}{2}-\frac{w_{y_{j, k}}^{i}+w_{y_{j, k+1}}^{i}}{2}\right) \cos \left(\theta_{m_{j, k}}^{i}\right) .
\end{aligned}
$$

We are now in a position to describe the discrete force equations.

The tangential force equation

$$
\begin{aligned}
R_{t j, k}^{i}= & \frac{f_{t j, k+1}^{i}-f_{t j, k}^{i}}{\Delta S_{j, k}^{i}}-\frac{\rho_{w} d \pi}{2} C_{t j}^{i}\left(v_{r t_{j, k+1 / 2}}^{i}\right)\left|v_{r t_{j, k+1 / 2}}^{i}\right|+ \\
& Q_{t}\left(s_{j, k+1 / 2}, t^{i-1}, v_{r t_{j, k+1 / 2}}^{i}, v_{r n}^{i}{ }_{j, k+1 / 2}^{i}\right) .
\end{aligned}
$$

The function $Q_{t}$ describes the DCU drag. It depends explicitly on $s$ because the DCUs are only at a few locations along the streamer. It depends explicitly on the time because the control angles are updated at each shot; these control angles will change the forces produced by the DCU and, hence, the shape of the streamer at the next shotpoint. The 
drag given by a DCU between two nodes is given by equation (26) using the velocities at the midpoints between the node, $Q_{t}$ is then given by dividing the force by the distance between the nodes.

The normal force equation

$$
\begin{aligned}
R_{n j, k}^{i}= & \frac{f_{t_{j, k+1}^{i}}^{i}+f_{t j, k}^{i}}{2} \frac{\theta_{j, k+1}^{i}-\theta_{j, k}^{i}}{\Delta S_{j, k}^{i}}-\frac{\rho_{w} d}{2} C_{n_{j}}^{i}\left(v_{r n_{j, k+1 / 2}}^{i}\right)\left|v_{r n_{j, k+1 / 2}}^{i}\right|+ \\
& Q_{n}\left(s_{j, k+1 / 2}, t^{i-1}, v_{r t_{j, k+1 / 2}^{i}}^{i}, v_{r n_{j, k+1 / 2}}^{i}\right) .
\end{aligned}
$$

The function, $Q_{n}$, describes the DCU horizontal steering force. If there is significant lateral steering applied then there may be a significant change in angle before and after the DCU. Because the DCU is only about $1 \mathrm{~m}$ long and the distance between nodes may be around $70 \mathrm{~m}$, the estimated drag across the streamer is adjusted to account for the change in angles. Based on the described setup, the approximate ${ }^{3}$ location of the DCU between the nodes is known. Therefore, it is assumed that the drag before the DCU is caused by the velocities and angle at the front node; the DCU forces and cross-flow drag is caused by the midpoint angles and velocities and, after the DCU, the drag is caused by the velocities and angle at the rear node.

Currents smooth along the streamers

As the position data have errors, that will lead to errors in the curvature, the first key assumption required to estimate the currents is that they are smooth in space. For a single streamer, this means that the currents are smooth along the streamer

$$
\begin{aligned}
R_{s x_{j, k}}^{i} & =\frac{w_{x_{j, k+1}^{i}}^{i}-w_{x_{j, k}^{i}}^{i}}{\Delta S_{j, k}^{i}}, \\
R_{s y_{j, k}}^{i} & =\frac{w_{y_{j, k+1}^{i}}^{i}-w_{y_{j, k}^{i}}^{i}}{\Delta S_{j, k}^{i}} .
\end{aligned}
$$

Currents close to the ADCP average

The vessel takes approximately one hour to travel the length of the streamers. During this time the ADCP on the vessel records the currents; therefore, this record provides the best prior information we have on the currents in the region the streamers occupy. As we expect the currents to vary in time and space, we assume that the mean recorded value of the ADCP currents, over the previous hour, gives the best prior estimate of the currents along the entire length of the streamers. Let us denote these means using $w_{a d c p}$. The standard deviation of this record gives an estimate of the expected variability in the currents, and is denoted by $\sigma_{a d c p}$. The ADCP residual equations are

$$
\begin{gathered}
R_{a d c p_{x j, k}^{i}}^{i}=w_{x j, k}^{i}-w_{a d c p_{x}}{ }^{i}, \\
R_{a d c p_{y_{j, k}}{ }^{i}}=w_{y_{j, k}}^{i}-w_{a d c p_{y}}{ }^{i} .
\end{gathered}
$$

\footnotetext{
${ }^{3}$ The streamer may have extended slightly and the streamer may have a slightly vertical profile.
} 
- Streamer position equations

The position (possibly processed) data contain errors. These lead to errors in the curvature, which, in turn, lead to errors in the inferred currents. Therefore, in the cost function, the positions are treated as unknowns that are constrained to be close to the data. These errors are correlated as they are calculated through the GPS positions on the floats and the acoustic ranges; however, to save computational cost, one may choose to pretend that this is not the case. Instead, one assumes that the position errors are independent of each other (assuming that there is just a small amount of error is sufficient to obtain smooth currents). If this simplifying assumption is made, the residual equations are

$$
\begin{gathered}
R_{x j, k}^{i}=x_{j, k}^{i}-\tilde{x}_{j, k}^{i}, \\
R_{y_{j, k}^{i}}^{i}=y_{j, k}^{i}-\tilde{y}_{j, k}^{i},
\end{gathered}
$$

where a tilde denotes the data.

- Streamer velocity equations

The reason for treating the streamer velocities as unknowns is because, when processing the data, to remove the noise as discussed in $\S 3.3$, the small jumps in the velocity when the DCUs suddenly provide lateral steering, are removed. The sudden small change in velocity is smoothed out - corrupting the velocity before and after the steering is turned on. Hence, the velocities contain errors. Because the $x$ and $y$ velocities of each node were processed separately in exactly the same way, it seems appropriate to treat the errors of the processed velocities as independent. Let us assume that the velocities are close to the processed values, so, the velocity residuals are

$$
\begin{aligned}
& R_{v_{x}{ }^{i}, k}^{i}=v_{x j, k}^{i}-\tilde{v}_{x j, k}^{i}, \\
& R_{v_{y j, k}}^{i}=v_{y_{j, k}}^{i}-\tilde{v}_{y_{j, k}}^{i},
\end{aligned}
$$

where a tilde denotes the processed velocities that are input into the cost function.

Currents are smooth in time in the earth reference frame

Despite treating the velocities as unknowns, when inferring the currents, there remain problems when the DCUs are providing lateral steering (see §4.3). Therefore, to attempt to overcome this problem let us assume that the ocean currents are only changing slowly in the earth reference frame. Because the streamer is moving, the time derivative of a variable, $w$, described in terms of the arc-length, $s$, and time, $t$, is given by

$$
\begin{aligned}
\frac{D w(s, t)}{D t}= & \frac{\partial w(x(s, t), y(s, t), t)}{\partial t}+v_{x}(s, t) \frac{\partial w(x(s, t), y(s, t), t)}{\partial x}+ \\
& +v_{y}(s, t) \frac{\partial w(x(s, t), y(s, t), t)}{\partial y}
\end{aligned}
$$

Hence, the time derivative of $w$ in the earth reference frame is

$$
\begin{aligned}
\frac{\partial w}{\partial t} & =\frac{D w}{D t}-v_{x} \frac{\partial w}{\partial x}-v_{y} \frac{\partial w}{\partial y}, \\
& \approx \frac{D w}{D t}-v_{t} \frac{\partial w}{\partial s}
\end{aligned}
$$


where the approximation is due to neglecting the normal velocity of the cable because it is small and, if considering the currents along a single streamer only, it is not possible to calculate the rate of change in the currents across the streamer. The discrete version of equation (43) forms a set of residual equations

$$
\begin{aligned}
& R_{x t_{j, k}^{i}}^{i}=v_{t_{j, k+1 / 2}^{j}} \frac{w_{x_{j, k+1}^{i}}^{i}-w_{x_{j, k}^{i}}^{i}}{\Delta S_{j, k}^{i}}-\frac{w_{x_{j, k+1 / 2}^{i}}^{i}-w_{x_{j, k+1 / 2}^{i-1}}}{\Delta t^{i-1}}, \\
& R_{y t_{j, k}}^{i}=v_{t_{j, k+1 / 2}^{j}}^{j} \frac{w_{y_{j, k+1}^{i}}^{i}-w_{y_{j, k}}^{i}}{\Delta S_{j, k}^{i}}-\frac{w_{y_{j, k+1 / 2}^{i}}^{i}-w_{y_{j, k+1 / 2}}^{i-1}}{\Delta t^{i-1}} .
\end{aligned}
$$

These residuals include the inferred currents at the previous shot time. Therefore, if these residual equations are included in the cost function, it is no longer possible to infer the currents at one shotpoint independently of the other shotpoints.

- The head constraints

The boundary conditions at the head of the streamer are that the tension at the head of the streamer should match the recorded tension (this is the only direct measure of the tangential currents along each streamer), allowing for drag caused by floats, miniwings and DCUs between the first node and the tow adapter where the tension is recorded. Let $\tilde{T}_{j}^{i}$ denote the recorded tension on each streamer at each shotpoint, and let $\Delta H_{j}$ denote the distance between the first node and where the tension is recorded (obtained from the towing configuration / survey design), then the tension residual is

$$
\begin{aligned}
R_{\text {tension } j}^{\text {head }}= & \tilde{T}_{j}^{i}-f_{t_{j, 1}^{i}}^{i}+\frac{\rho_{w} d \pi}{2} C_{t j}^{i} \Delta H_{j} v_{r t_{j, 1}}^{i}\left|v_{r t j, 1}^{i}\right|+ \\
& +\frac{T_{L j}+M_{j}}{2.5722^{2}} v_{r t_{j, 1}}^{i} \sqrt{v_{r t}^{i} t_{j, 1}^{i}+v_{r n}^{i}}-Q_{t, 1}\left(t^{i-1}, v_{r t} \underset{j, 1}{i}, v_{r n j, 1}^{i}\right) .
\end{aligned}
$$

The function, $Q_{t}$, describes the drag caused by a DCU just before the first node. If there is no miniwing on a streamer, then $M_{j}=0$, and if there is no front float $T_{L j}=0$.

The other constraint is that the currents at the head of the streamer should not be too far from the currents recorded on the ADCP on the vessel. Because the vessel is about $500 \mathrm{~m}$ in front of the first node on each streamer, the actual condition used is that the currents are close to the mean recorded value of the ADCP currents, over the previous $200 \mathrm{~s}$, denoted by $w_{\text {adcp } 200}$.

$$
\begin{aligned}
R_{\text {adcp } p_{x j}}^{\text {head }^{i}}=w_{x_{j, 1}}{ }^{i}-w_{\text {adcp } 200_{x}}{ }^{i}, \\
R_{\text {adcp }_{y_{j}} \text { head }^{i}}=w_{y_{j, 1}}^{i}-w_{\text {adcp } 200_{y}}{ }^{i} .
\end{aligned}
$$

- The tail constraint

The tail constraint is a discrete form of the tail buoy drag parameterization

$$
R_{t a i l j}^{i}:=f_{t j, K}^{i}+\frac{T_{L}}{2.5722^{2}} v_{r t}^{i}{ }_{j, K}^{i} \sqrt{v_{r t_{j, K}^{i}}^{i}+v_{r n}^{i}{ }_{j, K}^{i}}
$$

- Parameter assumptions

The force equations and boundary conditions depend on the drag coefficients and parameters for the tail buoy, front float, and miniwing drags. The values for these parameters 
are not certain; therefore, they are treated as unknowns, at each time step, constrained to be close to the prior estimates (denoted with a tilde),

$$
\begin{gathered}
R_{C_{t j}}{ }^{i}=C_{t j}^{i}-\tilde{C}_{t j}, \\
R_{C_{n j}}{ }^{i}=C_{n j}{ }^{i}-\tilde{C}_{n j}, \\
R_{M_{j}}{ }^{i}=M_{j}^{i}-\tilde{M}_{j}, \\
R_{T_{L} j}{ }^{i}=T_{L j}^{i}-\tilde{T}_{L} .
\end{gathered}
$$

- Possible extra constraints

Small tension perturbation

By constraining the currents to be close to the average recorded by the ADCP, the tension should remain close to the initial tension guess; however, if there are no ADCP data then the tension must be constrained to be close to the initial guess for the tension. This guess can be a linear guess using the recorded tension at the head of the streamer and the tail buoy assumption. Provided the inferred tension does not move far from this guess, it should be possible to infer the normal currents.

Kinematic equations

As we are considering the streamer velocity as an unknown that is close to the processed value, it may be necessary to add additional constraints to the problem. The most appropriate constraints on the streamer velocities are discrete forms of the kinematic equations (1) and (2). These equations are derived using the assumption that the streamer is inextensible; however, in the data, the length of the streamer varies by a few meters over the data set. This is either due to the fact that the streamer shape is actually three-dimensional (it does not quite lying in a horizontal plane), or due to the streamer stretching. To date, I have not found using these equations to be helpful in the inverse method.

\subsubsection{The coupling residuals.}

- Currents smooth across the streamers

Having assumed that the currents are smooth along the streamers, when treating multiple streamers, we should, also, assume that the currents are smooth in the crossline direction

$$
\begin{aligned}
R_{c x_{j, k}}^{i} & =\frac{w_{x j+1, k}^{i}-w_{x_{j, k}^{i}}^{i}}{\delta c_{j, k}^{i}}, \\
R_{c y_{j, k}^{i}}^{i} & =\frac{w_{y_{j+1, k}^{i}}^{i}-w_{y_{j, k}}^{i}}{\delta c_{j, k}^{i}} .
\end{aligned}
$$

- Currents are horizontally divergence free

Without tension measurements along the streamer, the key assumption to infer the 
inline currents is that the currents are approximately horizontally divergence-free,

$$
\begin{aligned}
R_{D i v_{j, k}^{i}}^{i}= & \frac{1}{2}\left(\begin{array}{c}
w_{x_{j, k+1}^{i}}^{i}+w_{x_{j, k}}^{i} \\
w_{y_{j, k+1}}^{i}+w_{y_{j, k}}^{i}
\end{array}\right) \cdot\left(\begin{array}{c}
-\Delta y_{j, k}^{i} \\
\Delta x_{j, k}^{i}
\end{array}\right)+\frac{1}{2}\left(\begin{array}{c}
w_{x_{j, k+1}^{i}}^{i}+w_{x_{j+1, k+1}^{i}}^{i} \\
w_{y_{j, k+1}}^{i}+w_{y_{j+1, k+1}^{i}}^{i}
\end{array}\right) \cdot\left(\begin{array}{c}
-\delta y_{j, k+1}^{i} \\
\delta x_{j, k+1}^{i}
\end{array}\right)+ \\
& \frac{1}{2}\left(\begin{array}{c}
w_{x_{j+1, k}^{i}}^{i}+w_{x_{j+1, k+1}^{i}}^{i} \\
w_{y_{j+1, k}}^{i}+w_{y_{j+1, k+1}^{i}}^{i}
\end{array}\right) \cdot\left(\begin{array}{c}
\Delta y_{j+1, k}^{i} \\
-\Delta x_{j+1, k}^{i}
\end{array}\right)+\frac{1}{2}\left(\begin{array}{c}
w_{x_{j, k}^{i}}^{i}+w_{x_{j+1, k}^{i}}^{i} \\
w_{y_{j, k}}^{i}+w_{y_{j+1, k}}^{i}
\end{array}\right) \cdot\left(\begin{array}{c}
\delta y_{j, k}^{i} \\
-\delta x_{j, k}^{i}
\end{array}\right) .
\end{aligned}
$$

- Acoustic network equations

Errors in the streamer curvature and velocity are due to errors in the hydrophone positions. Therefore, to obtain smooth currents, the position data are treated as unknowns in the cost function that are close to their recorded values. Because the positions are calculated from an acoustic network, the errors in the positions are correlated. To ensure that the errors are correctly correlated, the acoustic network equations are mimicked in the inverse scheme following code provided by Ken Welker (private communication, 2014).

The front floats and tail buoys provide a GPS location that has an error of around 1 $\mathrm{m}$; this anchors the locations of the head and tail of the streamer. The acoustic signal from each acoustic transmitter can be recorded by each acoustic receiver within a certain distance of the transmitter (that varies according to the conditions), providing a set of ranges (based on the travel time) with an error comparable to the GPS error. The positions of each transmitter and receiver are then calculated by solving a cost function, and the greatest uncertainty in the positions is at the centre of the array.

The data provided were not the raw range data, but, rather position data. We wish to treat the positions as unknowns so that we can obtain a smooth curvature estimate. Therefore, we wish to perturb the positions; this will change the ranges. Let us therefore calculate the ranges of the input data and treat these as the recorded range data from which the positions must be calculated. Because we do not have the front float locations, let us treat the first node on streamers with a front float, as if they had a GPS location (provided by the given position data). As has been mentioned, there is a noticeable change in angle to incorporate the tail buoy that is not thought to be due to currents at the depth of the streamers. So, to avoid artifacts in the inferred currents, we assume that the transmitter at the tail of the streamer has the GPS location (provided by the given position data); also, there is a transmitter at the tail buoy, so, we greatly increase the uncertainty of the tail buoy ranges. The effect of this will be to allow the currents to be smooth near the streamer array tail by adjusting the tail buoy location. The outputted tail buoy location will (probably) not be correct. Because the GPS locations do not actually occur where they would in the actual system we increase their uncertainty to $2.5 \mathrm{~m}$. To weight the acoustic network equations in the cost function, we divide each equation by the expected standard deviation of the error (whether that is a range or a GPS error). 


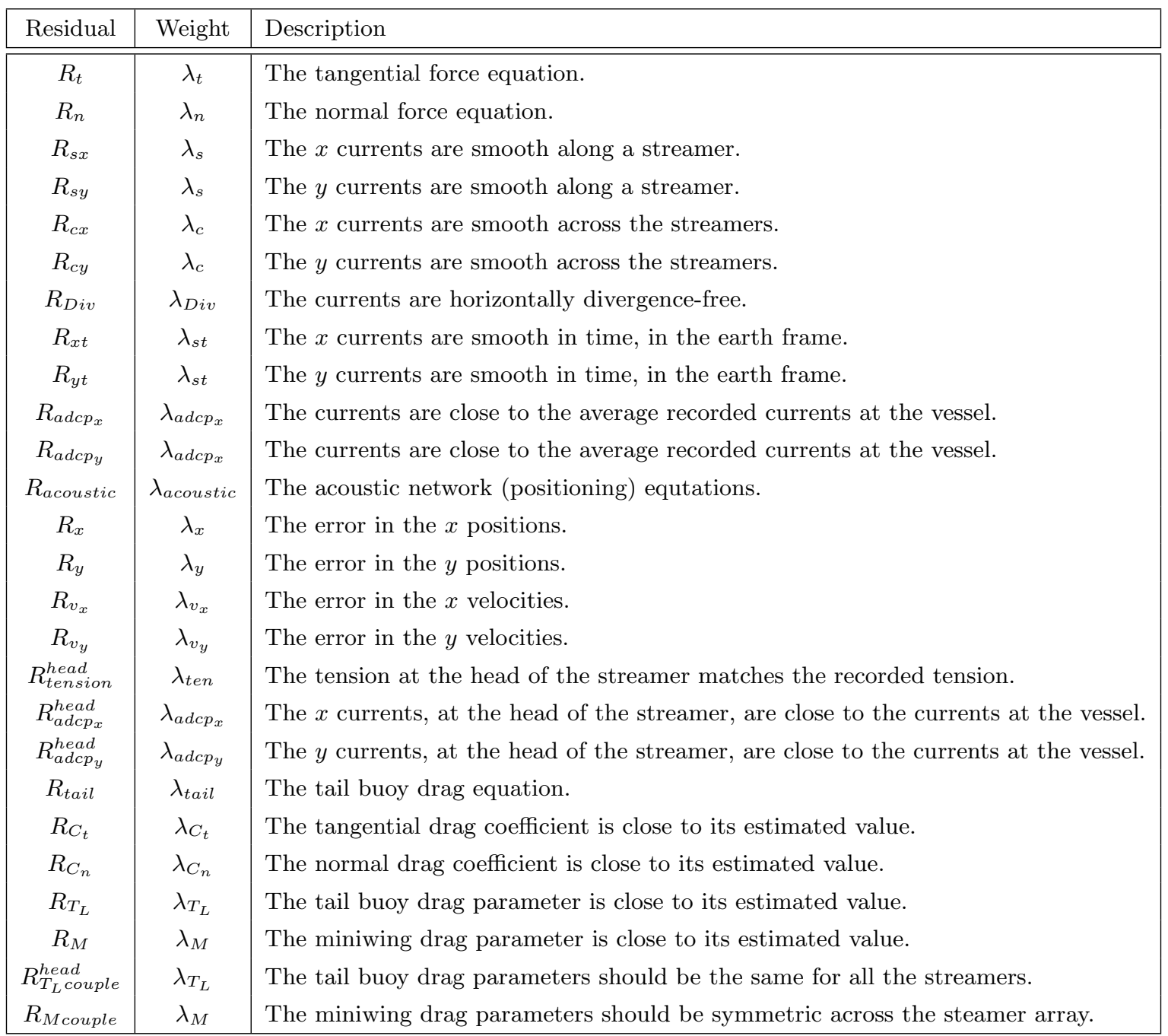

TABLE 2. The residuals, and their weights, that form the cost function.

Mimicking the acoustic solver produces a large number of equations, so, let us index the residual equations using $l$ and denote each equation by $R_{\text {acousticl }}$.

\section{- Parameter assumptions}

Becasue we are treating the parameters on the streamers as unknowns, when dealing with streamer arrays we can incorporate extra assumptions about the towing configuration to reduce the uncertainty. For example, although the miniwings have the same set control angle, due to the configuration, they may be providing slightly different drags, but as the towing configuration is symmetric, we should expect

$$
R_{\text {Mcouple }_{j}^{i}}^{i}=M_{j}^{i}-M_{12-j+1}^{i} .
$$

Similarly, the drag coefficients may be different on each streamer due to barnacles, but the drag caused by the front floats and tail buoys should be the same, so

$$
R_{T_{L} \text { couple }_{j}^{i}}=T_{L j}^{i}-T_{L j+1}^{i} .
$$

4.1.3. The cost function. The cost function, at each shot, is assembled from the residual equations, which are summarized in Table 2, 


$$
\begin{aligned}
& J^{i}\left(x, y, v_{x}, v_{y}, w_{x}, w_{y}, f_{t}, C_{t}, C_{n}, T_{L}, M\right)=\sum_{j=1}^{12} \sum_{k=1}^{K-1}\left(\lambda_{t}\left[R_{t j, k}^{i}\right]^{2}+\lambda_{n}\left[R_{n j, k}^{i}\right]^{2}+\lambda_{s}\left(\left[R_{s x j, k}^{i}\right]^{2}+\left[R_{s y_{j, k}}^{i}\right]^{2}\right)+\right.
\end{aligned}
$$

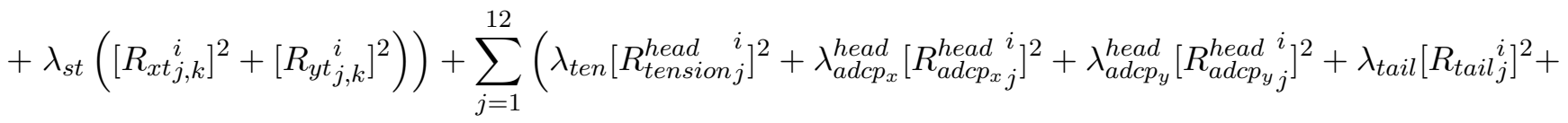

$$
\begin{aligned}
& \left.+\lambda_{C_{t}}\left[R_{C_{t j}}^{i}\right]^{2}+\lambda_{C_{n}}\left[R_{C_{n j}}^{i}\right]^{2}+\lambda_{T_{L}}\left[R_{T_{L} j}^{i}\right]^{2}+\lambda_{M}\left[R_{M_{j}}^{i}\right]^{2}\right)+\lambda_{\text {acoustic }} \sum_{l} R_{\text {acousticl }}^{i}+ \\
& \sum_{j=1}^{6} \lambda_{\text {Mcouple }}\left[R_{\text {Mcouple }_{j}^{i}}\right]^{2}+\sum_{j=1}^{11} \lambda_{T_{L} \text { couple }}\left[R_{T_{L} \text { couple }_{j}}\right]^{2}+ \\
& \sum_{j=1}^{11} \sum_{k=1}^{K} \lambda_{c}\left(\left[R_{c x j, k}^{i}\right]^{2}+\left[R_{c y j, k}^{i}\right]^{2}\right)+\sum_{j=1}^{11} \sum_{k=1}^{K-1} \lambda_{D i v}\left[R_{D i v j, k}^{i}\right]^{2}+ \\
& \sum_{j=1}^{12} \sum_{k=1}^{K}\left(\lambda_{{a d c p_{x}}}\left[R_{a d c p_{x j, k}}^{i}\right]^{2}+\lambda_{a d c p_{y}}\left[R_{a d c p_{y_{j, k}}{ }^{i}}\right]^{2}+\lambda_{x}\left[R_{x j, k}^{i}\right]^{2}+\lambda_{y}\left[R_{y_{j, k}}^{i}\right]^{2}+\lambda_{v_{x}}\left[R_{v_{x} j, k}^{i}\right]^{2}+\lambda_{v_{y}}\left[R_{v_{y j, k}}^{i}\right]^{2}\right),
\end{aligned}
$$

where the $\lambda$ s are weights that we are free to choose.

4.1.4. Choosing the weights. The main focus of this paper is to demonstrate that it is feasible to infer the ocean currents from the shapes of seismic streamers. The main difficulty of doing this is correctly choosing the smoothness and divergence weights in the cost function. We, therefore, must fix the other weights ${ }^{4}$ in the cost function. It seems best to base the weights on the expected errors in the different residuals.

For simplicity, let us assume that there is very little error in the drag coefficient estimates; let us assume that the errors have a standard deviation of $1 \%$ of the mean prior values. So, appropriate weights are

$$
\lambda_{C_{t}}=\left(\frac{100}{\tilde{C}_{t}}\right)^{2}, \quad \lambda_{C_{n}}=\left(\frac{100}{\tilde{C}_{n}}\right)^{2}, \quad \lambda_{T_{L}}=\left(\frac{100}{\tilde{T}_{L}}\right)^{2}, \quad \text { and } \quad \lambda_{M}=\left(\frac{100}{\tilde{M}}\right)^{2} .
$$

Let us put large weights on the symmetric assumptions so,

$$
\lambda_{\text {Mcouple }}=\left(\frac{10^{6}}{\tilde{M}}\right)^{2}, \quad \text { and } \quad \lambda_{T_{L} \text { couple }}=\left(\frac{10^{6}}{\tilde{T}_{L}}\right)^{2} .
$$

The author suspects that, by essentially fixing the unknown parameters that have errors, this will require a greater degree of smoothness in the inferred currents than would have been the case if the unknown parameters were known exactly.

The currents are inferred from the model equations. Therefore, we wish to give these a large weight and treat everything else as a form of regularization. Because the discretization is first order in space (it would be second order if the nodes were equally spaced), we use the order of

\footnotetext{
${ }^{4}$ Note that the variables and, hence, these weights, were scaled in the cost function used by the author. The values of $\lambda_{s}, \lambda_{D i v}$ and $\lambda_{s t}$ discussed later in this paper correspond to the scaled equations.
} 
the discretization error to weight the equations

$$
\lambda_{t}=\left(\frac{L}{\Delta s}\right)^{2}, \quad \lambda_{n}=\left(\frac{L}{\Delta s}\right)^{2}
$$

where $\Delta s$ is the average/representative step sized used and $L$ is the average length of the streamers.

How to weight the prior information from the ADCP data is not clear. The ADCP data provide a record of the currents in time, which we have interpreted as along a line in space because the currents are only changing slowly in time. We wish to infer the currents over an area, so if there is a significant shear in the currents across the streamer spread then placing too high a weight on the ADCP data may bias the solution against detecting this. Therefore let us use ${ }^{5}$

$$
\lambda_{a d c p_{x}}=\left(\frac{1}{{\sigma_{a d c p_{x}}}^{i}}\right), \quad \text { and } \quad \lambda_{a d c p_{y}}=\left(\frac{1}{{\sigma_{a d c p_{y}}}^{i}}\right) .
$$

In fact, it is still possible to obtain plausible inferred currents choosing $\lambda_{a d c p_{x}}=\lambda_{a d c p_{y}}=0$. However, because there are problems inferring the currents when there is lateral steering, it seems wise to use the ADCP data to help constrain the currents. Because the head of each streamer is around $500 \mathrm{~m}$ behind the vessel, the weights for the currents at the head of the streamers are weighted in terms of the smoothness weight

$$
\lambda_{\text {adcp }}^{\text {head }}=\lambda_{s}\left(\frac{1}{500 \sigma_{\text {adcp } 200_{x}}{ }^{i}}\right), \quad \text { and } \quad \lambda_{\text {adcp }}^{\text {head }}=\lambda_{s}\left(\frac{1}{500 \sigma_{\text {adcp } 200_{y}}{ }^{i}}\right) .
$$

If we choose to imitate the acoustic network $\lambda_{\text {acoustic }}=1$ and $\lambda_{x}=\lambda_{y}=0$. If we do not choose to imitate the acoustic network in the cost function, then $\lambda_{\text {acoustic }}=0$ and to give the positions freedom to move independently (so as to provide smooth curvature and hence currents) we set $\sigma_{x}=0.1 \mathrm{~m}$ and $\sigma_{y}=0.5 \mathrm{~m}$, so

$$
\lambda_{x}=\left(\frac{1}{\sigma_{x}^{i}}\right)^{2}, \quad \text { and } \quad \lambda_{y}=\left(\frac{1}{\sigma_{y}^{i}}\right)^{2} .
$$

We do not know the error in the processed velocities, but we assume that when the DCUs are steering, there may be an error in the currents of the order of centimeters per second; thus we initially assume $\sigma_{v_{x}}=\sigma_{v_{y}}=0.01$ and, so

$$
\lambda_{v_{x}}=\left(\frac{1}{\sigma_{v_{x}}}\right)^{2}, \text { and } \quad \lambda_{v_{y}}=\left(\frac{1}{\sigma_{v_{y}}}\right)^{2} .
$$

The head and tail tension conditions are important as they provide information on the total drag along the streamer and, so, we give them large weights

$$
\lambda_{\text {ten }}=\left(\frac{1}{\sigma_{\text {ten }}^{2}}\right), \quad \lambda_{\text {tail }}=\left(\frac{1}{\sigma_{\text {tail }}^{2}}\right)
$$

\footnotetext{
${ }^{5}$ The most sensible choice would actually be the square of these values; however, when doing this, the inferred $x$ currents tended to never move far from the initial guess regardless of the value of $\lambda_{D i v}$. This could be because the boundary conditions and smoothness assumptions are already forcing the currents to be close to the ADCP values.
} 
where $\sigma_{\text {ten }}=10 \mathrm{~N}$ (because there is clearly some noise in the tension measurement, see Figure 2) and $\sigma_{\text {tail }}=1 \mathrm{~N}$.

The weight $\lambda_{s t}$ reflects how smooth we expect the currents to be in time. We do not have any prior knowledge about this and so we initially set $\lambda_{s t}=0$. This has the benefit that the currents are inferred independently at each shotpoint, allowing us to judge the plausibility of the inferred currents based on the appearance of any coherent structures in time. The reason for imposing that the currents are smooth in time is to help constrain the inferred currents when there are errors in streamer velocity due to a sudden change in the lateral steering. Clearly, if estimating the velocity of the streamer with lateral steering could be done accurately then $R_{x t}$ and $R_{y t}$ could be neglected from the cost function, and smoothness in time could be used solely as a criteria (see $\S 4.2$ and equation (68)) for judging whether plausible currents have been estimated.

The weight $\lambda_{s}$ reflects how smooth we expect the currents to be in space along the streamers. We do not have any prior knowledge about this and, so, we must find it by experimenting. How to do this is discussed in $\S 4.2$. The weight $\lambda_{c}$ reflects how smooth we expect the currents to be in space across the streamers. The most sensible value for this is that $\lambda_{c}=\lambda_{s}$, however one might wish to set $\lambda_{c}=0$ and then use smoothness in space across the streamers as a criteria for selecting $\lambda_{s}$ (see equation (69)).

Finally, we do not have any prior knowledge on how to choose $\lambda_{D i v}$ and so this too is found by experimentation.

4.1.5. Minimizing the cost function. The cost function was minimized using MATLAB's nonlinear least-squares solver lsqnonlin. This solver, when provided with an overdetermined system of residual equations and their Jacobian uses a trust-region-reflective algorithm (MathWorks 2014a).

4.2. Inferring the currents without lateral steering. There are two weights that have not yet been determined: $\lambda_{s}$ and $\lambda_{D i v}$. Appropriate values are found by attempting to estimate the currents (whilst imitating the acoustic network) with different choices of weights, in a portion of the data where there is no significant steering. The results of doing this are shown in Figures 8, 9,10 and 11. So what is the best choice of weights? In all the experiments, the currents at each time have been inferred independently (except for how the data were processed); therefore, any structures that occur in time provide a diagnostic tool. By assumption, currents in the ocean change slowly in time compared with the speed of the vessel; therefore, any coherent vertical structures in Figures 10 and 11 are likely to be physical structures. However, diagonal structures (moving from bottom right to top left) are features moving at the speed of the vessel and, so, are very unlikely to be physical (they are probably artifacts of how the data were acquired and processed) and, so, indicate that the smoothness weight is not large enough. If, however, the smoothness weight is increased too much, then the amplitude of any signal in the ocean currents is suppressed and the currents tend to a constant value along each streamer at each step. This suggests a criteria for choosing an appropriate smoothness weight (for a given divergence weight). Choose the minimum of a cost function formed by the time derivative of the 
inferred currents in the earth reference frame using (45), scaled by the inferred velocity range,

$$
J_{\text {TimeEarth }}\left(\lambda_{s} ; \lambda_{\text {Div }}\right)=\left(\frac{1}{\max _{i, j, k}\left(w_{y_{j, k}}^{i}\right)-\min _{i, j, k}\left(w_{y_{j, k}}^{i}\right)}\right)^{2} \sum_{i, j, k}\left[R_{y t_{j, k}^{i}}^{i}\right]^{2} .
$$

This cost function, for each choice of $\lambda_{\text {Div }}$, is shown in the right panel in Figure 12. This indicates that the best choice of $\lambda_{s}$ is between $10^{0}$ and $10^{1}$ (depending on the choice of $\lambda_{D i v}$ ), however, by eye, looking at Figures 10 and 8 some features of the currents are being smoothed out when $\lambda_{s}>10^{0}$ and so, because I do not wish to over-smooth any inferred currents, I think that a better choice for $\lambda_{s}$ may be found between $10^{-1}$ and $10^{0}$. This judgement is supported by the fact that when $\lambda_{s}=10^{1}$ none of the divergence weights produces a noticeable signal in $w_{x}$ in Figures 11 and 9. It also fits with the general observation that, as $\lambda_{s}$ increases, the required value of $\lambda_{\text {Div }}$ needed to see possible $x$ currents increases.

If $\lambda_{c}=0$ then the smoothness of the currents in space across the streamers can also be used to evaluate the choice of $\lambda_{s}$. In this case we would look for the minimum of the cost function

$$
J_{\text {across }}\left(\lambda_{s} ; \lambda_{D i v}\right)=\left(\frac{1}{\max _{i, j, k}\left(w_{y_{j, k}}^{i}\right)-\min _{i, j, k}\left(w_{y_{j, k}}^{i}\right)}\right)^{2} \sum_{i, j, k}\left[R_{c y_{j, k}^{i}}^{i}\right]^{2} .
$$

When $\lambda_{D i v=0}$ the inline currents cannot be inferred. The inferred inline currents remain close to the initial guess so as to satisfy the tension and ADCP data. The inline currents can only be inferred by increasing the divergence weight. When the weight is sufficiently large, coherent structures for $w_{x}$ appear in both the spatial (Figure 9) and the time domains (Figure 11). If $x$ currents are being inferred then, in the streamer frame, $\frac{\partial w_{x}}{\partial t}$ should be noticeably larger than when $\lambda_{\text {Div }}$ is not high enough and the currents are close to constant. This suggests another cost function

$$
J_{\text {TimeStreamer }}\left(\lambda_{D i v} ; \lambda_{s}\right)=\left(\frac{1}{\max _{i, j, k}\left(w_{x j, k}^{i}\right)-\min _{i, j, k}\left(w_{x j, k}^{i}\right)}\right)^{2} \sum_{i, j, k}\left[\frac{w_{x j, k}^{i}-w_{x j, k}^{i-1}}{\Delta t^{i-1}}\right]^{2},
$$

which, rather than the minimum, we seek where it suddenly jumps. In the middle panel of Figure 12 notice that this jump occurs later the greater the value of $\lambda_{s}$. When $\lambda_{s}=10^{-1}$ this change takes place when $\lambda_{\text {Div }}$ is between $10^{8}$ and $10^{9}$, and when $\lambda_{s}=10^{0}$ this change takes place when $\lambda_{\text {Div }}$ is between $10^{9}$ and $10^{10}$.

If the divergence weight is too high, then we suspect that the inferred $w_{y}$ will be noticeably perturbed from the $\lambda_{D i v}=0$ case (which we believe, provided the inferred $w_{x}$ stays close to the ADCP data, should be reasonably accurate). This is apparent in the first two columns of Figures 8 and 10 where the $\lambda_{D i v}=0$ (top row) solution is visibly different from the $\lambda_{D i v}=10^{11}$ (bottom row) solution. This difference is measured using

$$
J_{\text {change }}\left(\lambda_{\text {Div }} ; \lambda_{s}\right)=\sum_{i, j, k}\left[w_{y_{j, k}^{i}}^{i}\left(\lambda_{D i v} ; \lambda_{s}\right)-w_{y_{j, k}}^{i}\left(0 ; \lambda_{s}\right)\right]^{2} .
$$

The left panel of Figure 12 shows how this cost increases with $\lambda_{\text {Div }}$. It also shows that the greatest increases occur at approximately at the same time as the jump in $J_{\text {TimeStreamer }}$.

Using these cost functions and making subjective judgments, the author believes that the best range of weights is found for $\lambda_{s} \in\left(10^{-1}, 10^{0}\right)$ and $\lambda_{\text {Div }} \in\left(10^{8}, 10^{9}\right)$. The same experiment 


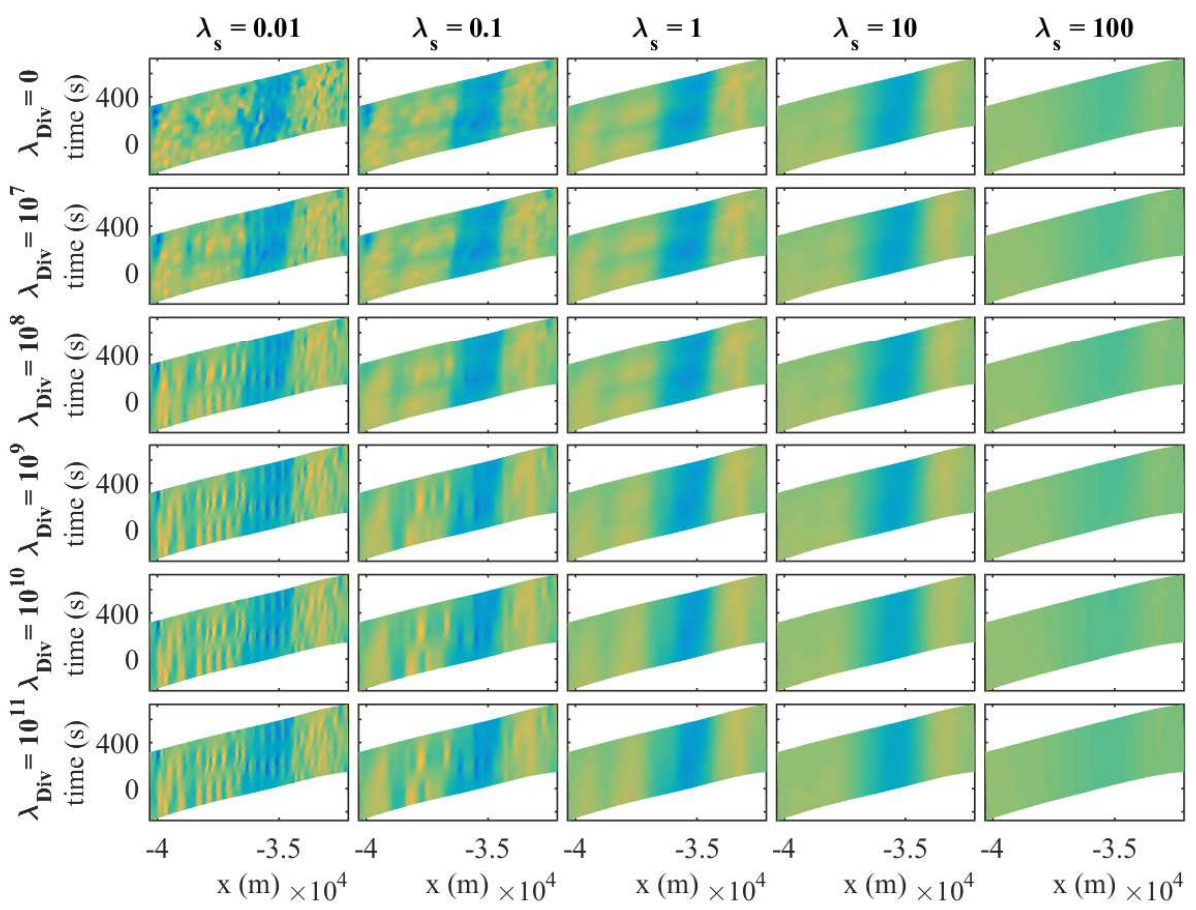

FiguRE 8. The inferred $y$ currents for different choices of smoothness and divergence weights. The colour scale is from $-0.0061 \mathrm{~m} / \mathrm{s}$ (blue) to $0.2819 \mathrm{~m} / \mathrm{s}$ (yellow): the minimum and maximum estimated values. For small $\lambda_{s}$, increasing $\lambda_{\text {Div }}$ pulls the inferred $y$ currents aways from the currents inferred without a divergence-free assumption.

can be repeated, focusing on this parameter region; however, it remains a subjective choice about how smooth the currents should be. Therefore, I shall use $\lambda_{s}=0.5$ and $\lambda_{D i v}=10^{10}$ when considering data with lateral steering (I have chosen the higher value of $\lambda_{D i v}$ to ensure that there is signal in $w_{x}$ ).

Before doing so, it is instructive to see how much the velocities and positions have changed from their input values. These changes are shown in Figures 15, 16, 13 and 14. There are features in the changes in the velocities that are moving back along the streamer, this suggests that some signal in the currents is being lost and is being attributed to errors in the processed velocities. This is consistent with the changes in velocity increasing as $\lambda_{s}$ increases. Figure 14 clearly shows that, if the smoothness weight is too high then the adjustments to the position data become unrealistic, and so, looking at the changes in the position data, provides another means of restricting the choice of weights. 


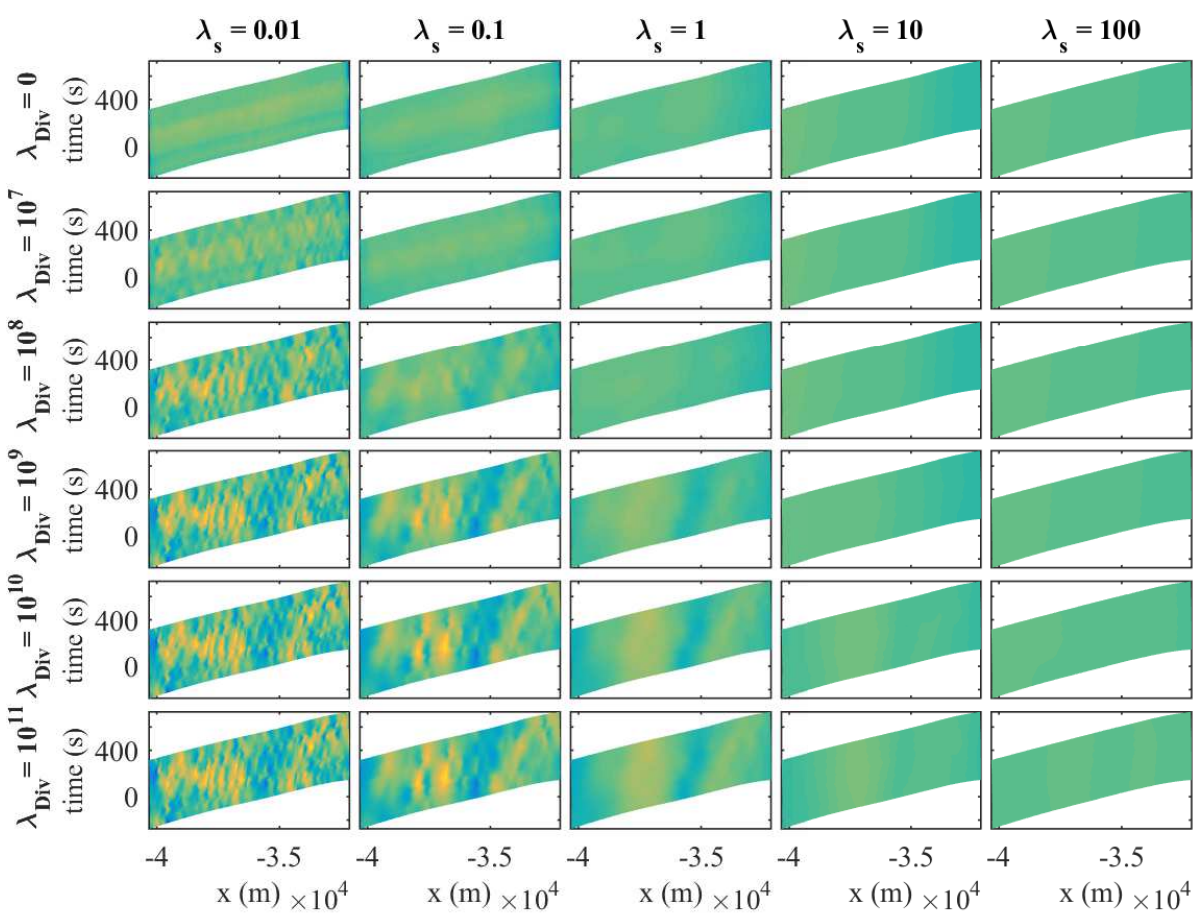

FiguRE 9. The inferred $x$ currents for different choices of smoothness and divergence weights. The colour scale is from $-0.0607 \mathrm{~m} / \mathrm{s}$ (blue) to $0.1957 \mathrm{~m} / \mathrm{s}$ (yellow): the minimum and maximum estimated values. As $\lambda_{s}$ increases, $\lambda_{D i v}$ must increase to see a signal in the $x$ currents. 


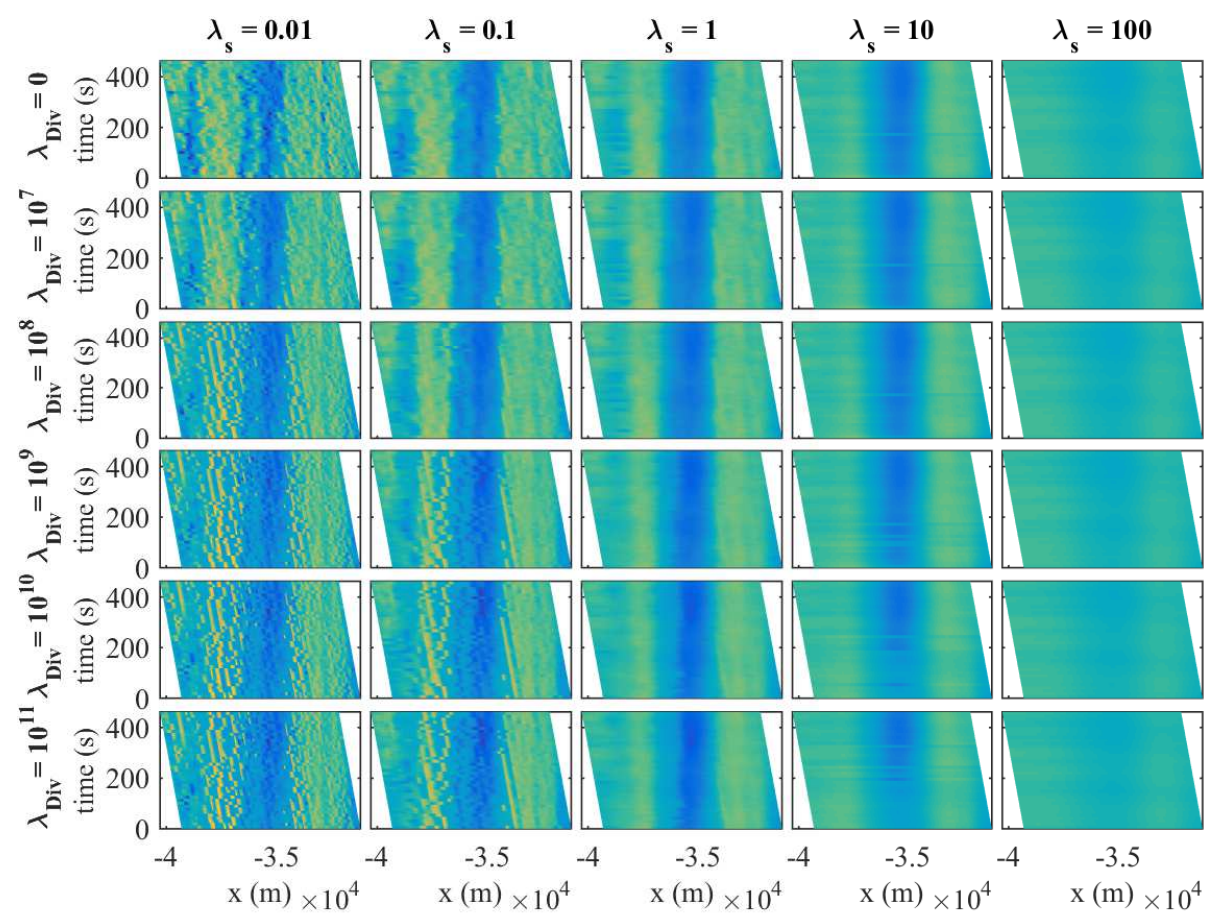

Figure 10. The inferred $y$ currents for different choices of smoothness and divergence weights, for Streamer 6 at successive time steps. The colour scale is from $0.0539 \mathrm{~m} / \mathrm{s}$ (blue) to $0.2922 \mathrm{~m} / \mathrm{s}$ (yellow): the minimum and maximum estimated values. For small $\lambda_{s}$, increasing $\lambda_{\text {Div }}$ pulls the inferred $y$ currents aways from the currents inferred without a divergence-free assumption. 


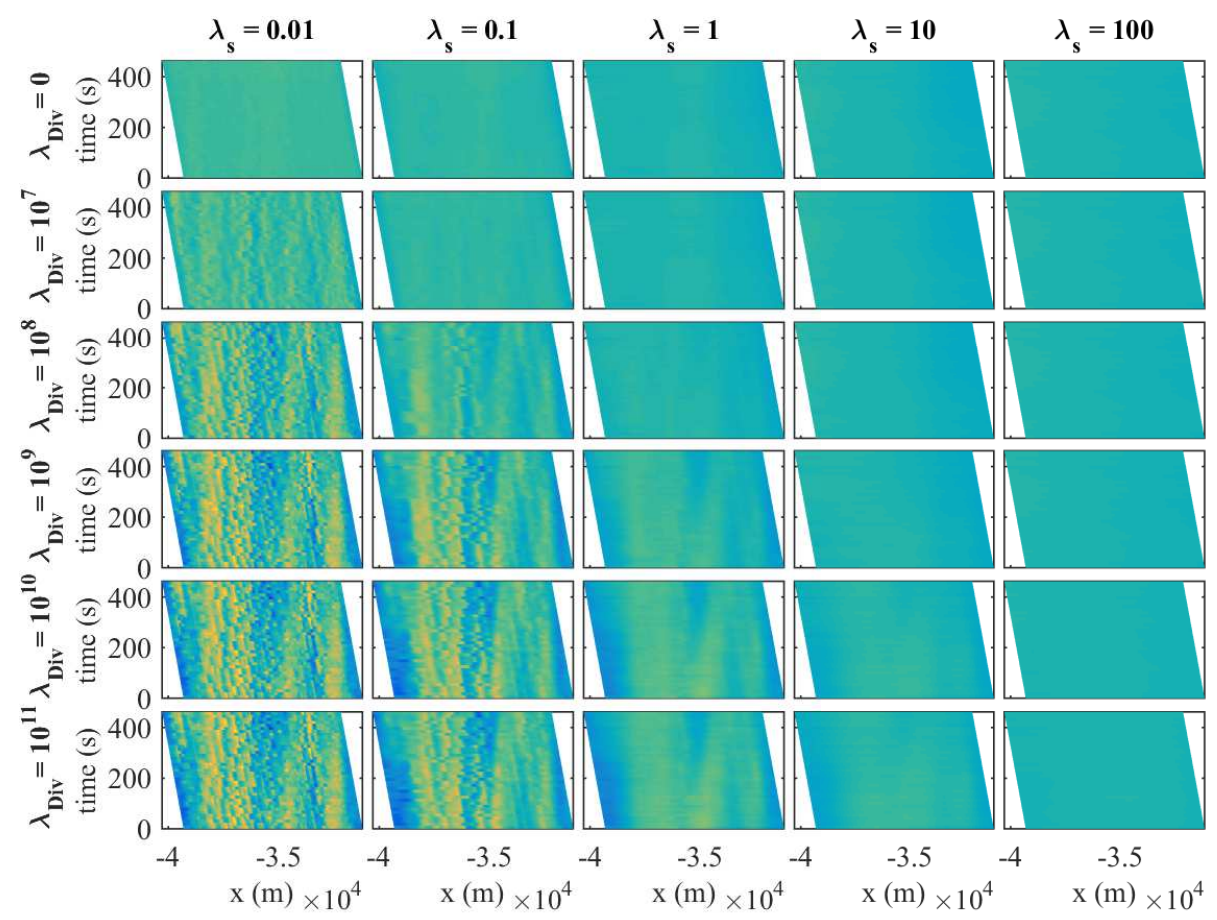

Figure 11. The inferred $x$ currents for different choices of smoothness and divergence weights, for Streamer 6 at successive time steps. The colour scale is from $-0.0460 \mathrm{~m} / \mathrm{s}$ (blue) to $0.2328 \mathrm{~m} / \mathrm{s}$ (yellow): the minimum and maximum estimated values. As $\lambda_{s}$ increases, $\lambda_{D i v}$ must increase to see a signal in the $x$ currents. 

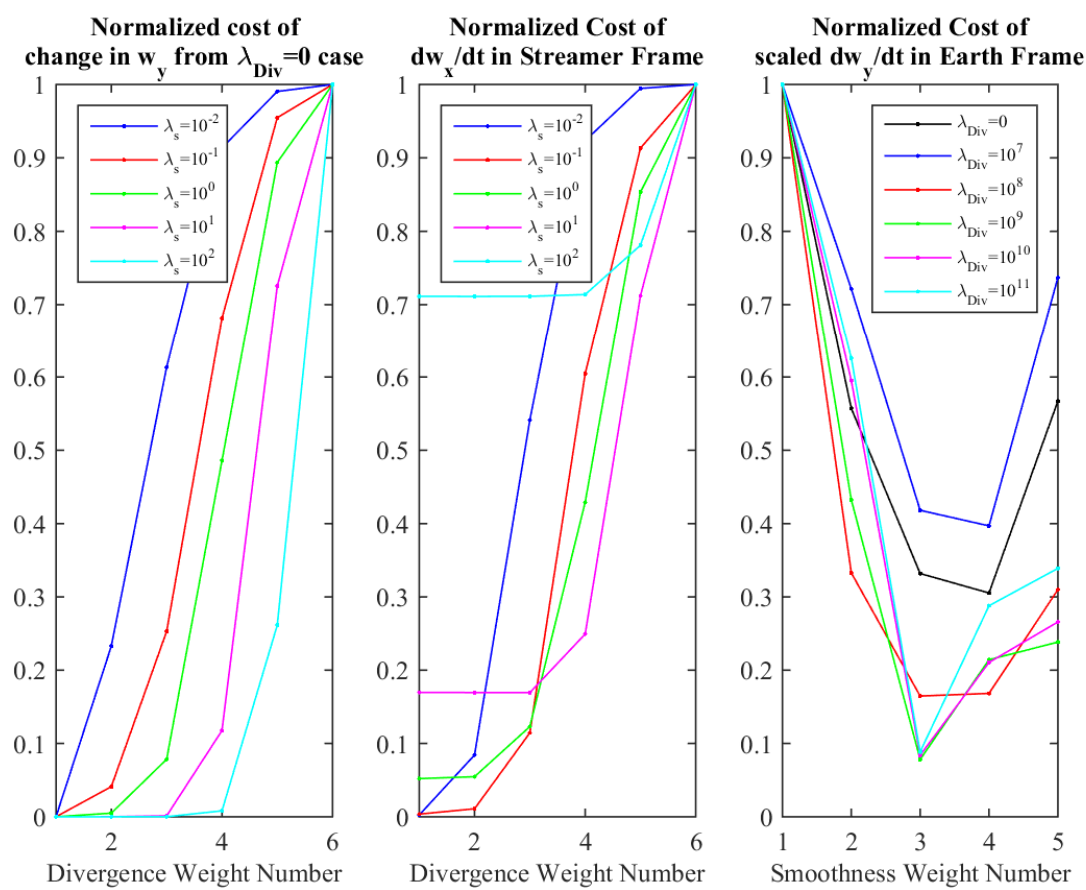

FiguRE 12. Normalized cost functions to help choose appropriate weights by fixing one of the weights. For a fixed value of $\lambda_{s}$ we seek the point where $\frac{\partial w_{x}}{\partial t}$ suddenly increases and where $w_{y}$ has not changed much from the $\lambda_{D i v=0}$ case. For a given $\lambda_{D i v}$ we seek the minimum of $\frac{\partial w_{y}}{\partial s}$ in the earth reference frame. 


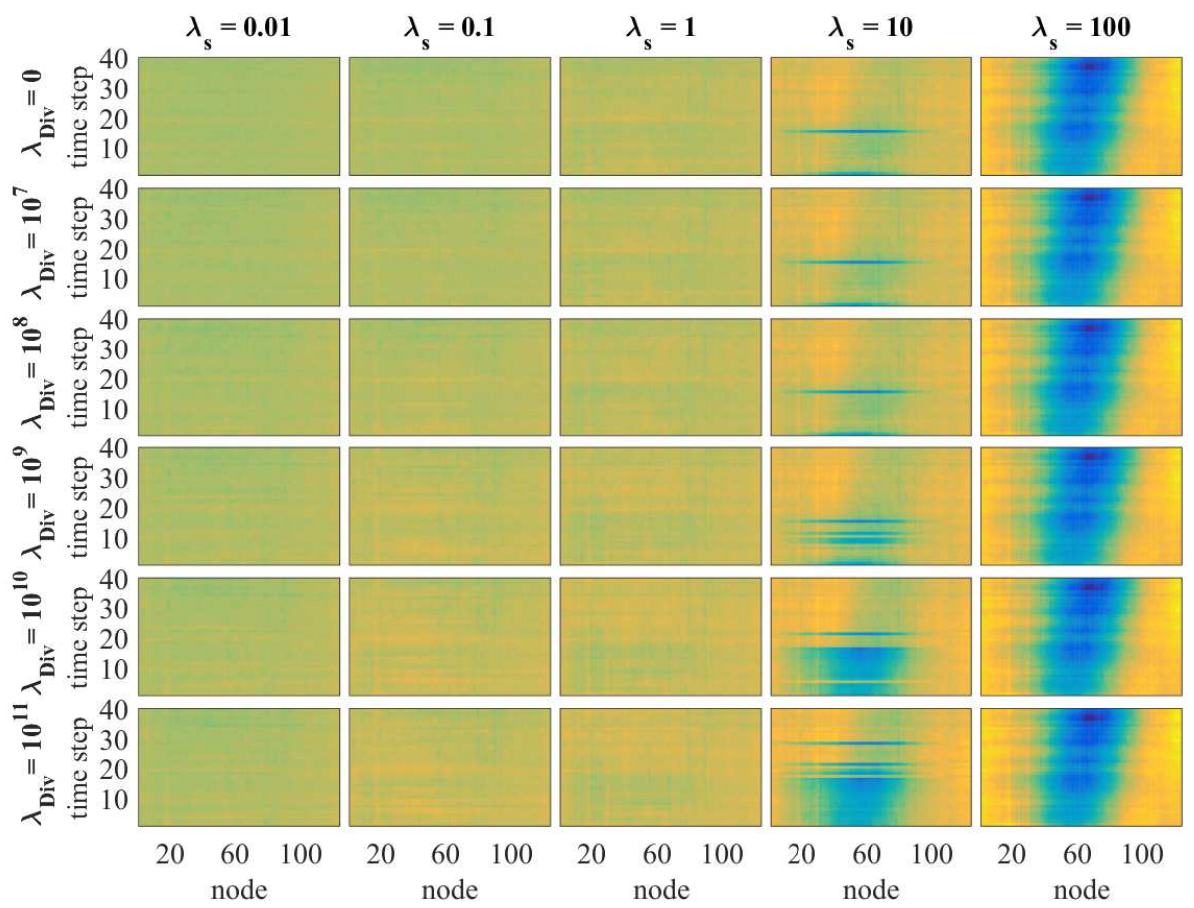

Figure 13. The change in the $x$ location of each node along Streamer 6 . The colour scale is from $-0.8841 \mathrm{~m}$ (blue) to $0.4475 \mathrm{~m}$ (yellow), which are the minimum and maximum changes in $x$. Note that when $\lambda_{s} \geq 10^{1}$ the change in $x$ moves down the streamer in time, suggesting that the smoothness weight is too high, and that curvature caused by currents is being flattened out. 


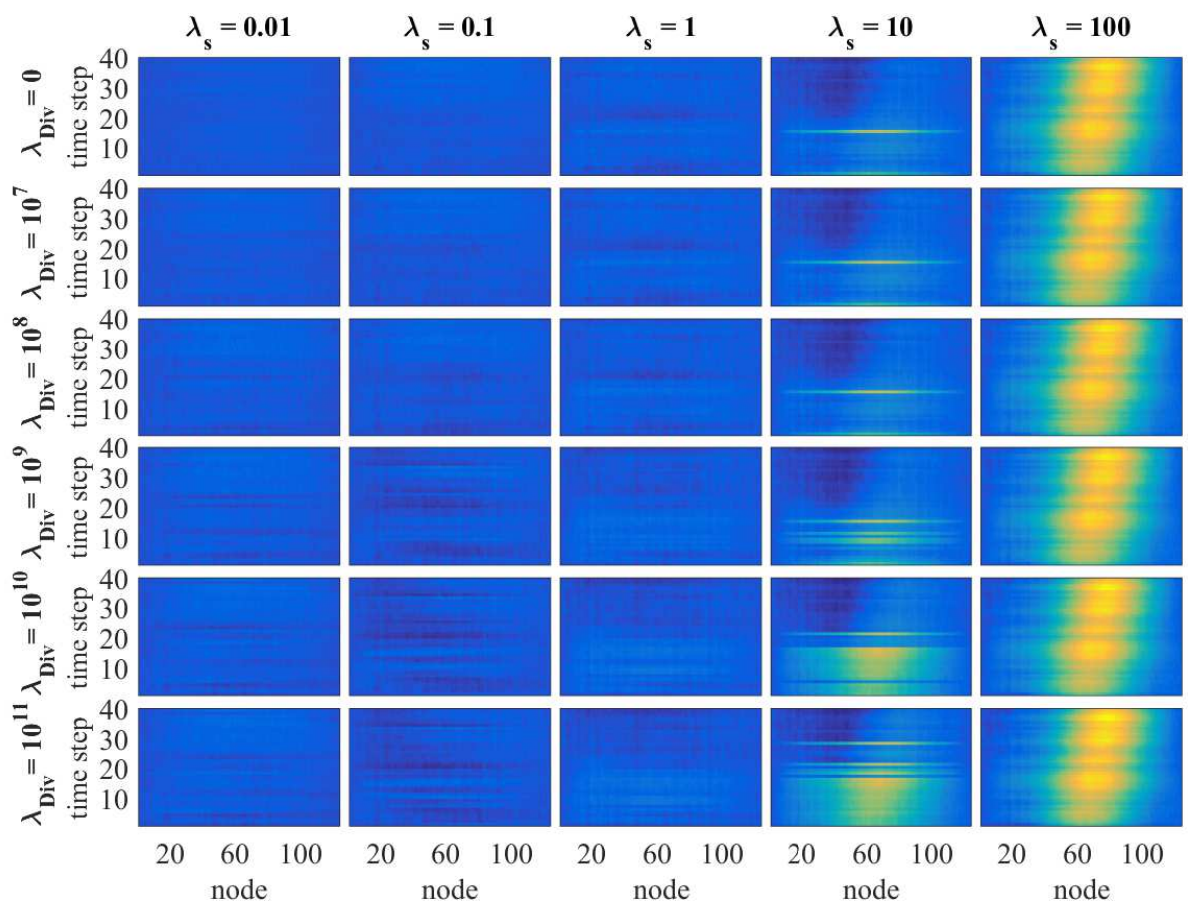

Figure 14. The change in the $y$ location of each node along Streamer 6 . The colour scale is from -2.0245 $\mathrm{m}$ (blue) to $19.2807 \mathrm{~m}$ (yellow), which are the minimum and maximum changes in $y$. Note that when $\lambda_{s} \geq 10^{1}$ the change in $y$ moves down the streamer in time, suggesting that the smoothness weight is too high, and that curvature caused by currents is being flattened out. 


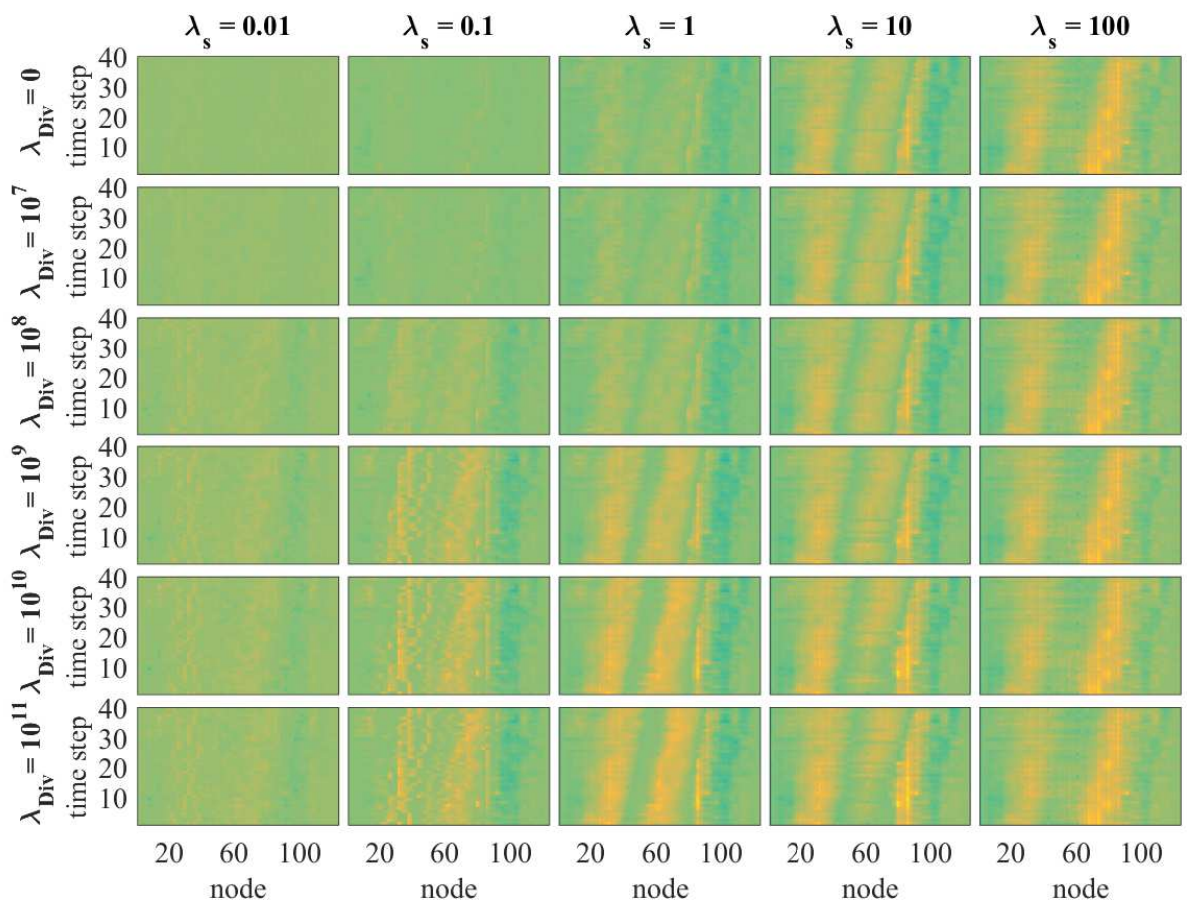

Figure 15. Change in $v_{x}$ from the input value for each node along Streamer 6 . The colour scale is from $-0.0021 \mathrm{~m} . \mathrm{s}^{-1}$ (blue) to $0.0011 \mathrm{~m} . \mathrm{s}^{-1}$ (yellow), which are the minimum and maximum changes in $v_{x}$. Note the features that move down the streamers, this suggests that signal is being lost in the currents and being attributed to velocity errors. 


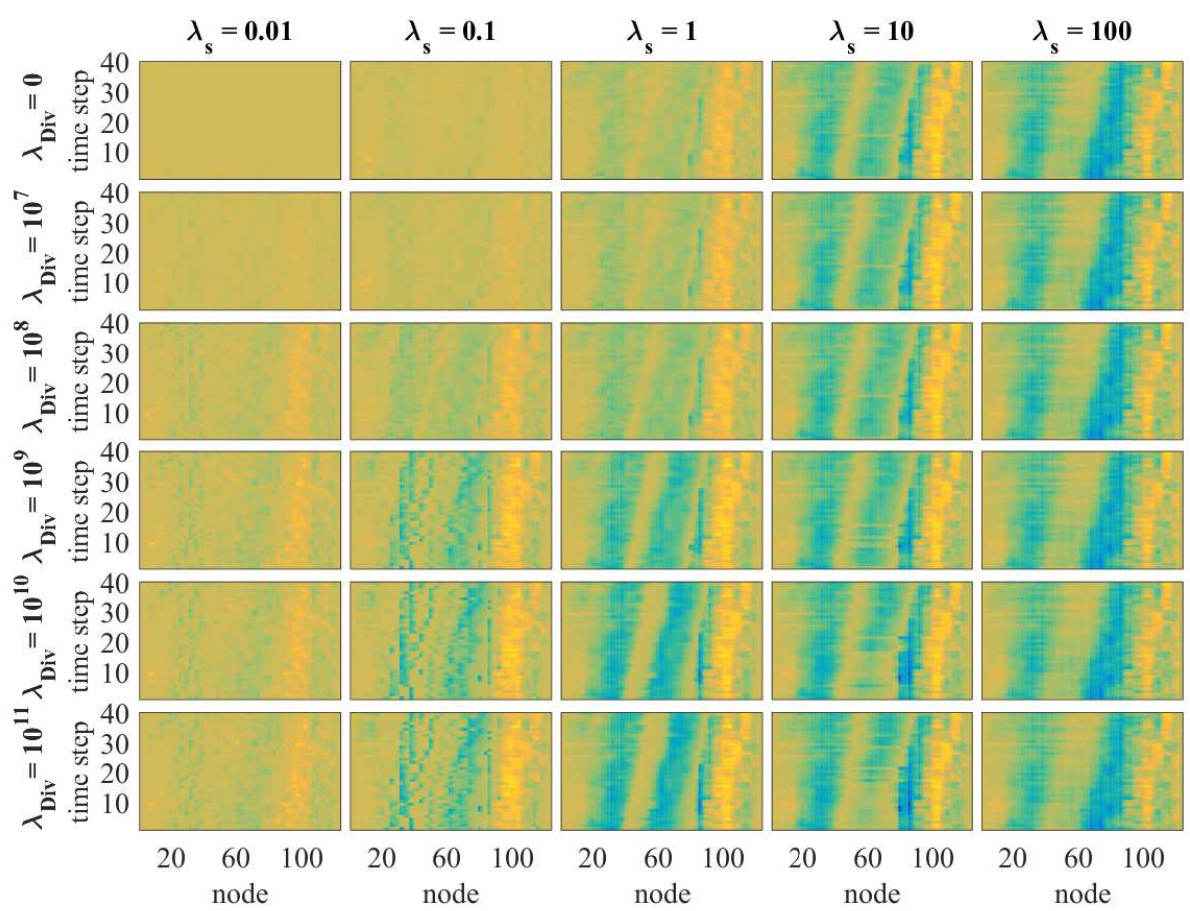

Figure 16. Change in $v_{y}$ from the input value for each node along Streamer 6 . The colour scale is from $-0.0255 \mathrm{~m} . \mathrm{s}^{-1}$ (blue) to $0.0089 \mathrm{~m} . \mathrm{s}^{-1}$ (yellow), which are the minimum and maximum changes in $v_{y}$. Note the features that move down the streamers, this suggests that signal is being lost in the currents and being attributed to velocity errors. 
4.3. Inferring the currents with lateral steering. Let us fix $\lambda_{s}=0.5$ and $\lambda_{\text {Div }}=10^{10}$ and now look at a region of data in which there is significant lateral steering: the middle three DCUs are instructed to provide maximum lateral steering. We now discover that when there is significant steering, artifacts appear in the inferred currents. I believe that these artifacts are caused by there being errors in the velocity introduced by the processing.

The streamer curvature informs us of the relative streamer velocity to that of the water. If there is an error in the normal (approximately $y$ ) velocity of the streamer, this will lead to an error in the inferred normal current, even if the relative velocity is correctly inferred. However, it is unlikely that the relative normal velocity of the streamer to the water is inferred correctly at the DCUs. This is because, if there is a large error in the normal velocity of the streamer, then in-order to obtain a correct relative normal velocity it will require a large change in the normal currents, which our smoothness and prior assumptions about the magnitude of the currents may not allow. Therefore, assuming the position data do not have much freedom to change and so affect the curvature, the normal force equation is made to balance by adjusting the DCU force estimate. This can be achieved through adjusting the speed of the streamer through the water; this largely affects the in-line currents, or through the angle of attack that the streamer makes with the water, this is primarily achieved through adjusting the cross-line current estimate.

To overcome this problem, we assume that the streamer velocity is an unknown in the cost function. The hope is that the jump in the normal streamer velocity, when lateral steering is applied, is restored. To aid this, we assume that the currents are smooth in time. The idea is that at the previous time step, the inferred currents away from the DCUs should be reasonably accurate, so, when the portion of streamer with the DCU reaches this location, the currents should not have changed much. Therefore, any large changes in the inferred relative velocity should be attributed to the velocity of the streamer rather than to changes in the currents. The danger in doing this is that the inferred currents at the head of the streamer (dependent on the head boundary condition) are simply advected along the streamer, and the information contained in the curvature is ignored. Therefore, let us investigate the effects of increasing our uncertainty in the processed velocities and the value of the smoothness in time weight, $\lambda_{s t}$.

The top row in each of Figures 17 to 22 show the results of not imposing any smoothness in time on the currents $\left(\lambda_{s t}=0\right)$. In Figure 17 there is a clear jump apparent in the inferred cross currents when the steering is turned on. The effect on the inferred $x$ currents is less clear; however, it is still visible as a small step in the currents in Figure 18. If the DCUs providing the steering are near the tail of the streamer, the effect is far more dramatic. I believe this is because, when the steering is near the centre of a streamer, the smoothness in space constraint aids in restoring the inline currents to realistic values downstream of the steering. When the steering is at the tail of the streamer there are no realistic inferred currents downstream of the steering to restrain the error in $w_{x}$ so the error is far worse.

The good news is that, by just increasing $\lambda_{s t}$ a small amount (looking at the second row in the figures, in which $\lambda_{s t}=0.2$ ) the worst effects of the steering are mitigated. Rather than the error in $w_{x}$ being smeared across the whole length of the streamer, the error in all the cases is restricted to the location of the DCUs. In fact, the local anomaly in $w_{x}$ is not noticeable when 
the assumed error in the velocities is $0.03 \mathrm{~m} / \mathrm{s}$ or greater (Figure 18), and is not noticeable in the final column of Figure 18. However, when the velocity error is assumed to be $0.01 \mathrm{~m} / \mathrm{s}$, the step in $w_{x}$ near the fins is visible for all values of $\lambda_{s t}$.

Removing artifacts in the inferred $y$ currents requires a greater degree of smoothness in time. Unlike the $x$ currents, the more problematic case is when the middle three fins are steering (Figure 17) rather than when the steering is at the tail of the streamers. This could be because, unfortunately, there appears to be a jump in the currents (before the steering is applied) that seems to coincide with the location of the central fins. Thus, the smoothness is space constraint seeks to match the currents either side of the fins, and has difficulty placing the jump in a consistent location. The best parameter choice for removing this jump is $\sigma_{v_{x}}=\sigma_{v_{y}}=0.05$ and $\lambda_{s t}=1$, yet the presence of the DCUs is still discernible. In the first two columns of Figure 17, after the initial jump when the DCUs start steering, there are features in the currents that move with the streamer suggesting that the assumed error in the currents is not large enough. In the middle column these features are barely visible once $\lambda_{s t}=0.6$, and in the final column they are barely visible once $\lambda_{s t}=0.2$.

Because we do not wish to lose too much signal in the currents by allowing too much freedom to change the velocities, or by imposing that the currents are smooth in time, it is probably best to choose weights so that (at the least) the artifacts caused by the steering are localized about the DCUs and not spread along the streamer. Beyond that, it is desirable that features in $w_{y}$ that move with the streamer when the DCUs are steering are eliminated. In our examples, this requires $\sigma_{v_{x}}=\sigma_{v_{y}} \geq 0.03$. However, imposing a value of $\lambda_{s t}$ great enough to entirely remove the jump in $w_{y}$ when the DCUs are turned on probably risks losing too much signal elsewhere. Therefore, in $\S 4.4$, when inferring the currents over the entire data, I use $\sigma_{v_{x}}=\sigma_{v_{y}}=0.03$ and $\lambda_{s t}=0.5$.

It is interesting to see the changes in the velocities that have been inferred. These are shown in Figures 19 and 20. For the weight values that eliminate the worst artifacts of the steering, there seems to be a change of about $0.01 \mathrm{~m} / \mathrm{s}$ in $v_{x}$ and $0.1 \mathrm{~m} / \mathrm{s}$ in $v_{y}$, when the DCUs start steering from the processed velocities. Similarly, looking at the changes in the position data, in Figures 21 and 22 is instructive. The more accurate we assume the velocity data to be, the worse the effect of imposing that the currents are smooth in time is on the position data is. This is the case in the bottom left plots in Figures 21 and 22; to minimise the cost functions, unrealistic perturbations to the position data have been enforced. 


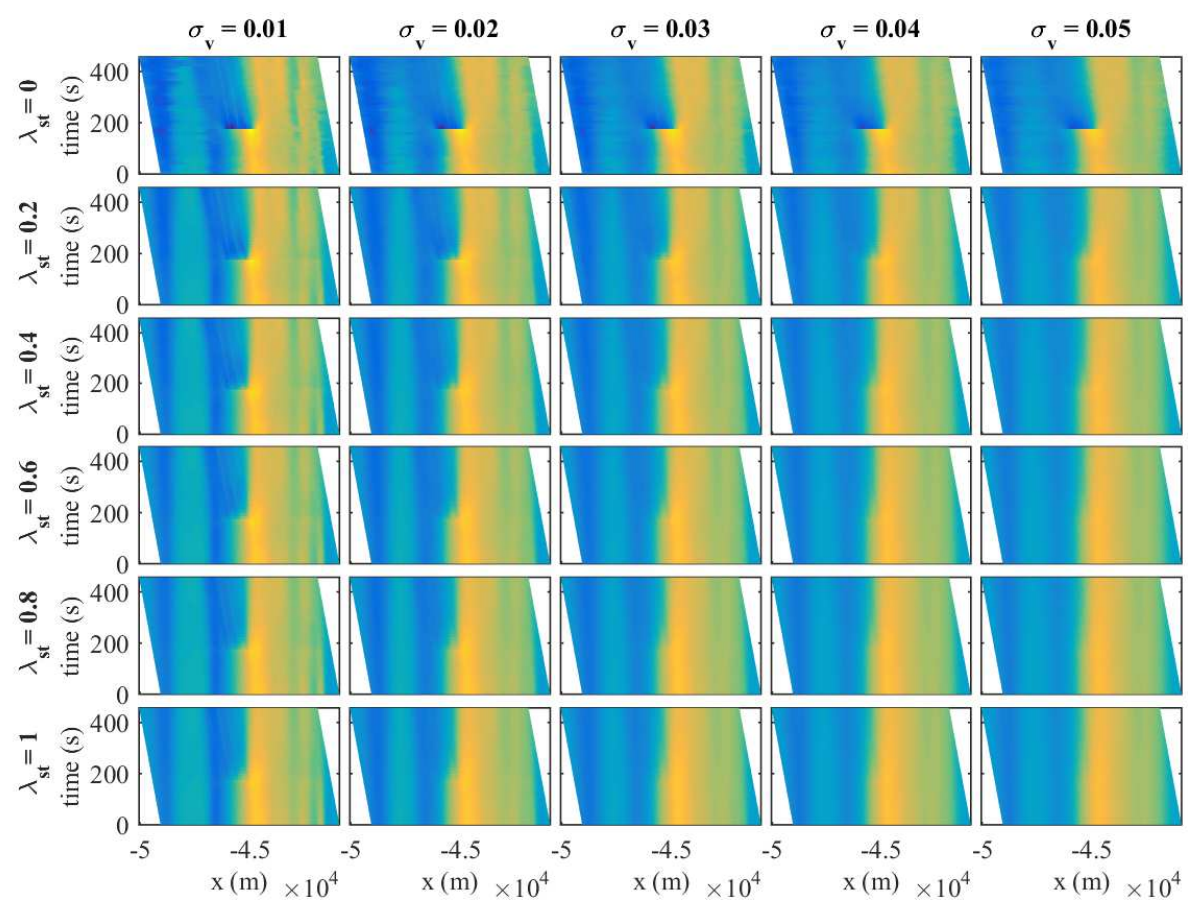

FiguRE 17. The inferred $y$ currents for different choices of velocity errors and smoothness in time weighting, for Streamer 6 at successive time steps. The colour scale is from $0.1147 \mathrm{~m} / \mathrm{s}$ (blue) to $0.3226 \mathrm{~m} / \mathrm{s}$ (yellow): the minimum and maximum estimated currents.

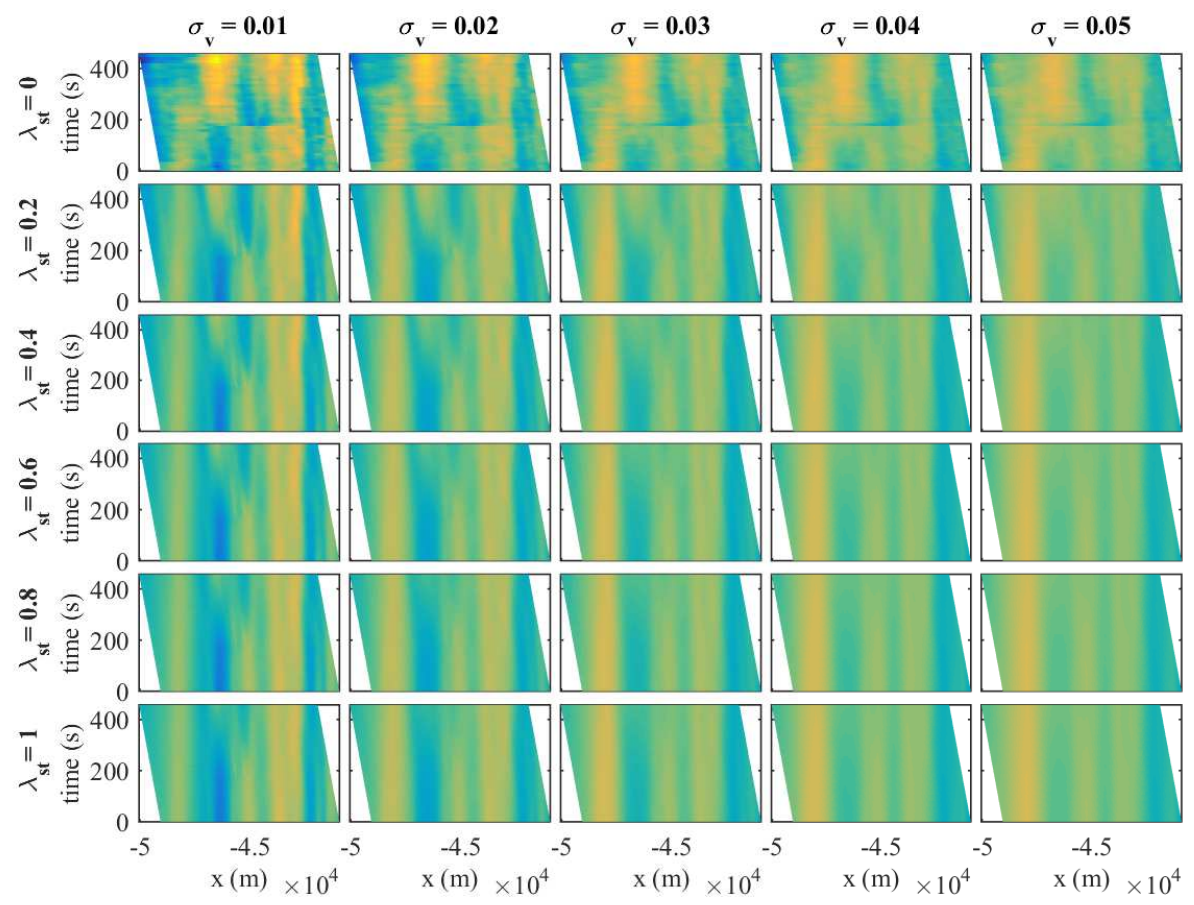

FiguRE 18. The inferred $x$ currents for different choices of velocity errors and smoothness in time weighting, for Streamer 6 at successive time steps. The colour scale is from $0.0299 \mathrm{~m} / \mathrm{s}$ (blue) to $0.1338 \mathrm{~m} / \mathrm{s}$ (yellow): the minimum and maximum estimated currents. 


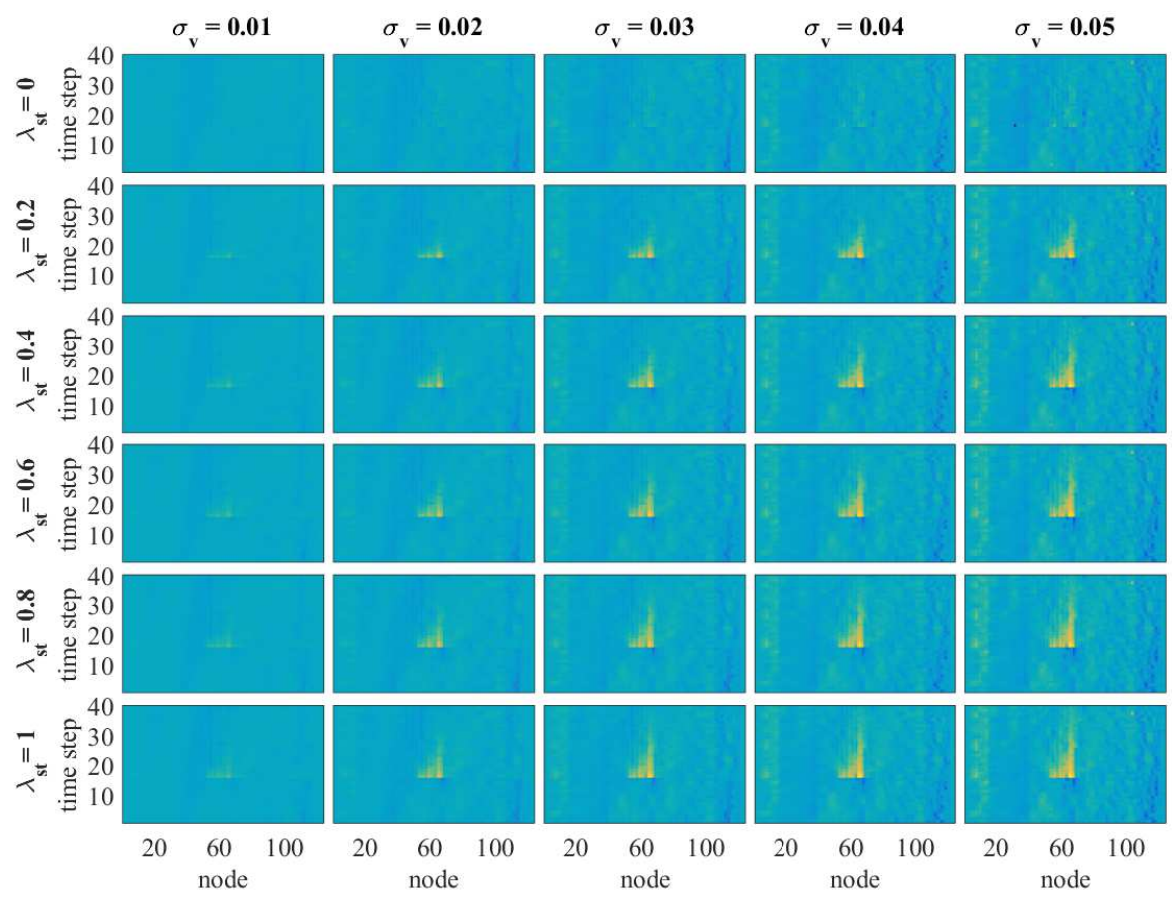

Figure 19. The change in $v_{y}$ from the input value, for different choices of velocity errors and smoothness in time weighting, for Streamer 6 at successive time steps. The colour scale is from $-0.1036 \mathrm{~m} . \mathrm{s}^{-1}$ (blue) to $0.1554 \mathrm{~m} . \mathrm{s}^{-1}$ (yellow), which are the minimum and maximum changes in $v_{y}$.

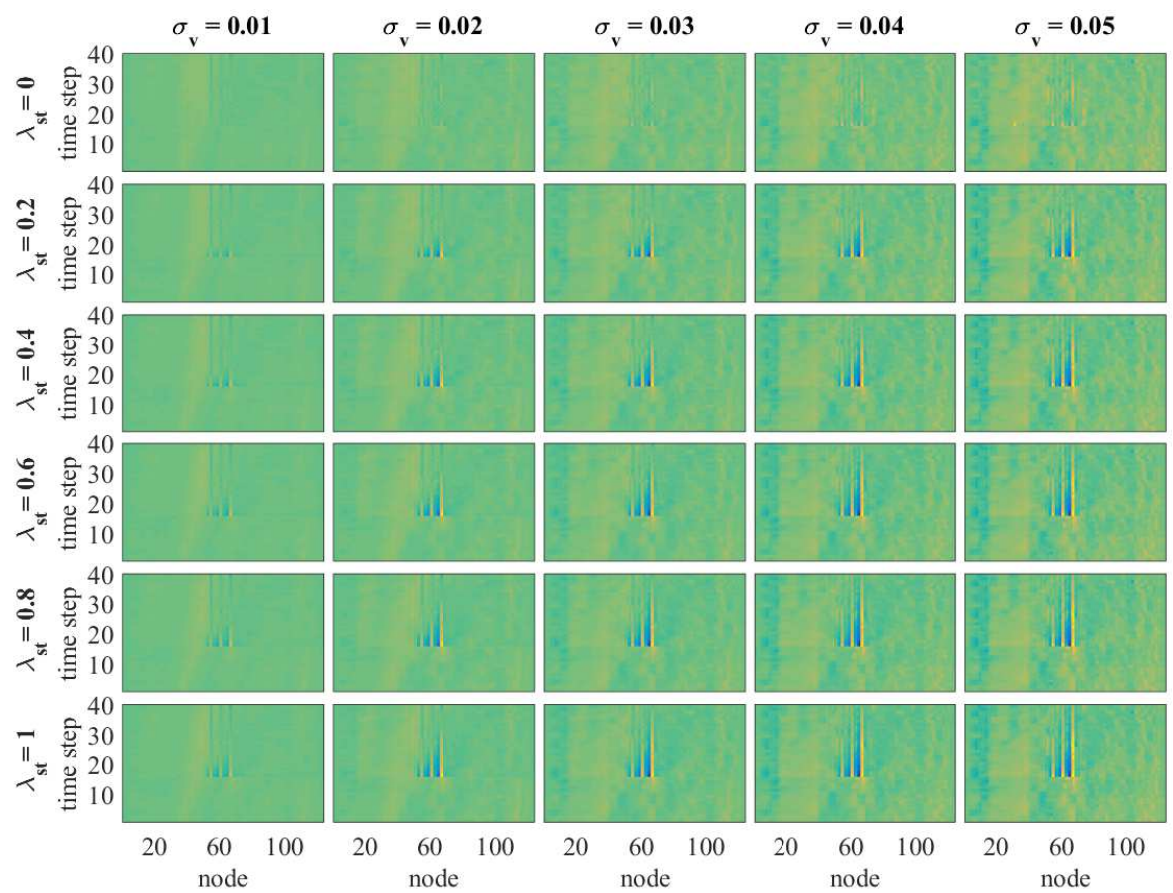

Figure 20. The change in $v_{x}$ from the input value, for different choices of velocity errors and smoothness in time weighting, for Streamer 6 at successive time steps. The colour scale is from $-0.0163 \mathrm{~m} . \mathrm{s}^{-1}$ (blue) to $0.0120 \mathrm{~m} . \mathrm{s}^{-1}$ (yellow), which are the minimum and maximum changes in $v_{x}$. 


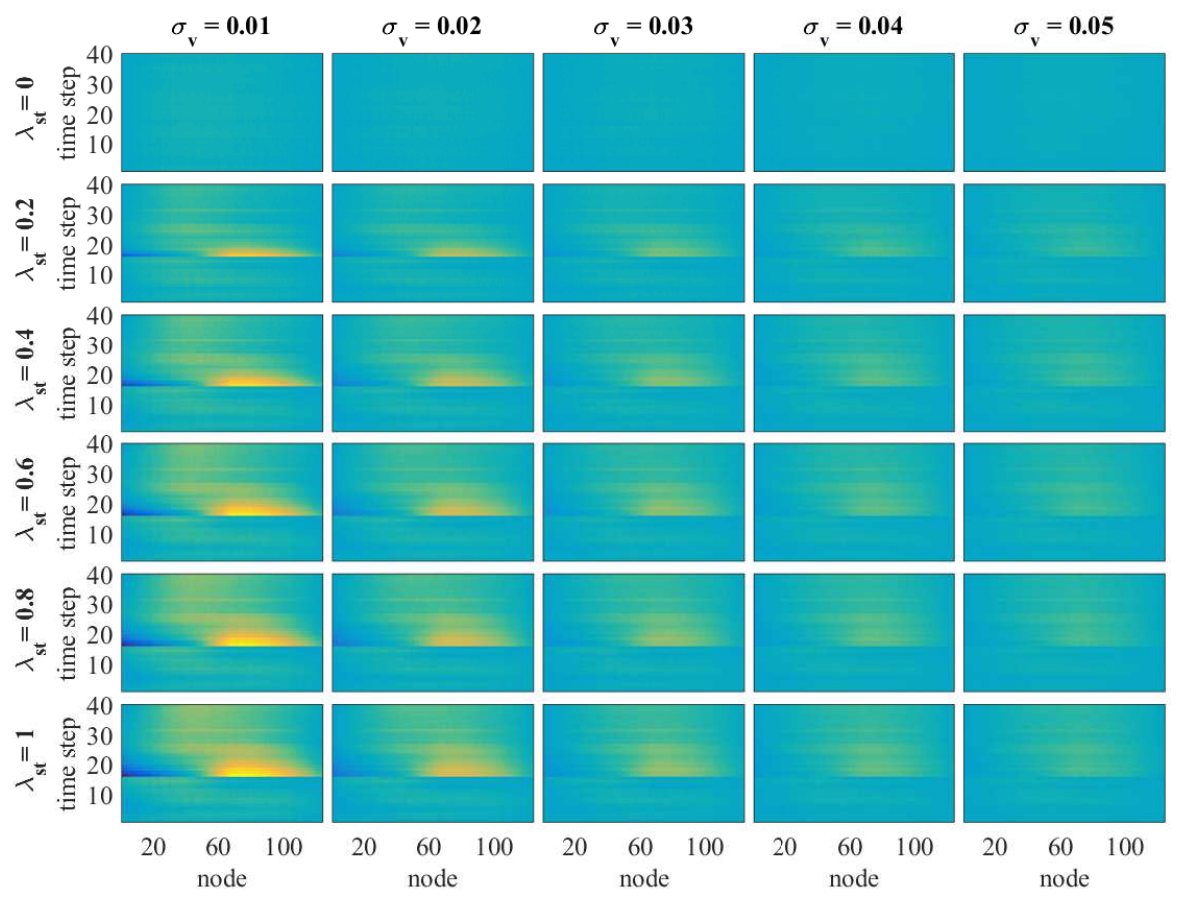

Figure 21. The change in $y$ from the input value, for different choices of velocity errors and smoothness in time weighting, for Streamer 6 at successive time steps. The colour scale is from $-18.9600 \mathrm{~m}$ (blue) to $30.5117 \mathrm{~m}$ (yellow), which are the minimum and maximum changes in $y$.

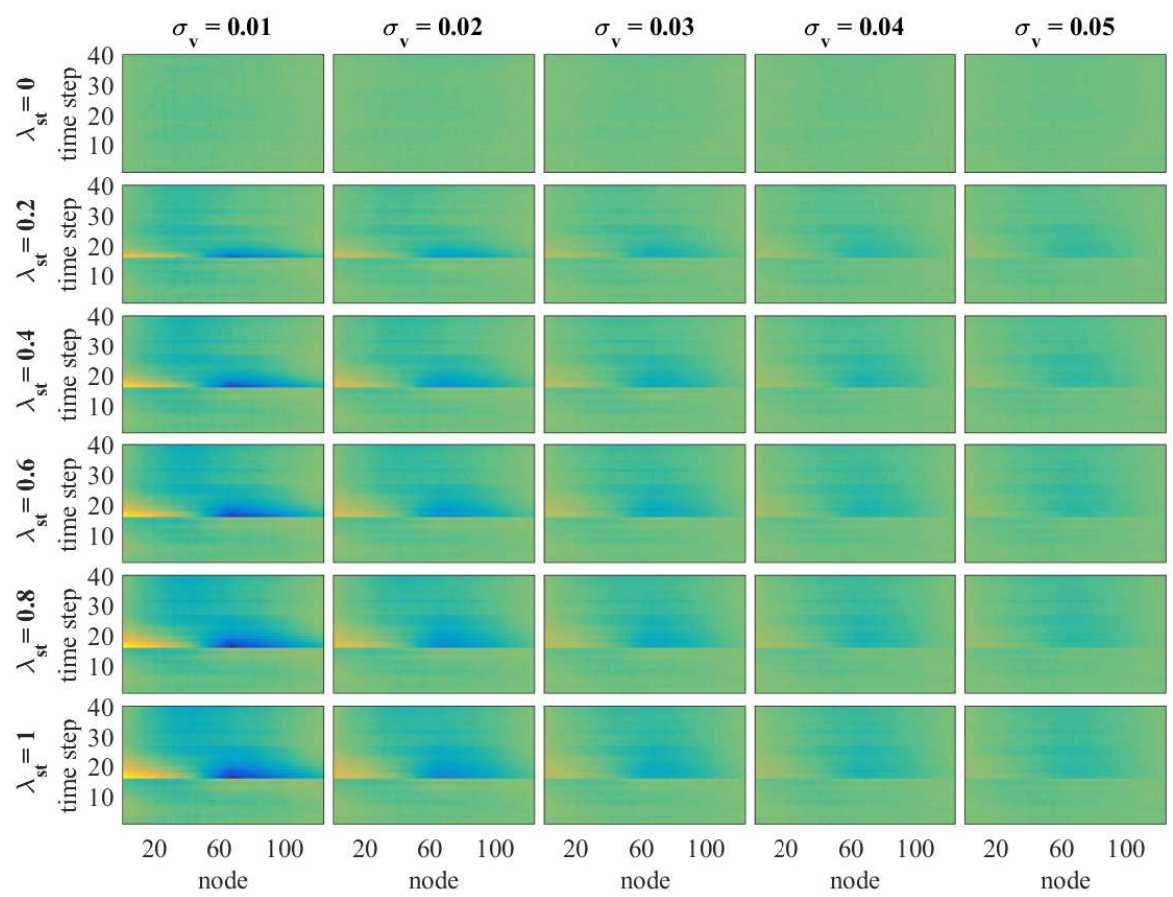

Figure 22. The change in $x$ from the input value, for different choices of velocity errors and smoothness in time weighting, for Streamer 6 at successive time steps. The colour scale is from $-3.1665 \mathrm{~m}$ (blue) to $2.2465 \mathrm{~m}$ (yellow), which are the minimum and maximum changes in $x$. 
4.4. Inferring the currents for all the data. Having performed experiments on small sections of the data, let us now attempt to infer the currents for all the available data. I have not seen a major difference between assuming that the position errors are independent and that they are correlated through the acoustic network; therefore, to reduce computational time, the acoustic network is not imitated and the position errors are treated as independent (i.e., $\lambda_{\text {acoustic }}=0$ ). The weights used in this section (based on our experimentation) are $\lambda_{s}=0.5$, $\lambda_{\text {Div }}=10^{10}, \lambda_{s t}=0.5$ and $\sigma_{v x}=\sigma_{v y}=0.03$.

Figure 23 shows the currents inferred along Streamer 6. The results seem to be plausible currents that are slowly varying in time. Imposing smoothness in time has not resulted in the currents being fixed at each location. However, there are some noticeable artifacts. At the very tail of the streamer, between 21 and 23 hours, there is a noticeable line, moving with the streamer, in the $y$ currents that is caused by lateral steering at the tail. In $\S 4.3$, we accepted this as a price for not over-smoothing the currents. Of more concern, is that there is a dip in the $y$ currents, moving with the centre of streamer, after the central three DCUs are turned on just after 26 hours. This was not apparent in the small section of data examined in $\S 4.3$. Therefore, it seems that we are still struggling to infer the currents when there is steering. Figure 29 show the inferred forces produced by the DCUs when the middle fins are turned on. It is noticeable that the inferred horizontal forces are much less than the recorded forces. This is partly due to the fact that the recorded forces are actually calculated from the DCU angles, assuming that the speed of the streamer through the water is $2.57 \mathrm{~m} / \mathrm{s}(5 \mathrm{knots})$; whereas, the true speed is less than this. The speed through the water is predominantly the difference between $v_{x}$ and $w_{x}$, which, as there are no apparent artifacts in $w_{x}$, is probably reasonably accurate. However, errors in the relative $y$ velocity between the streamers and the current will affect the angle of attack that the DCU makes, and, so, may well, be affecting the estimated force.

Despite our lack of faith in the estimated currents when there is steering, we can compare the inferred currents with the ADCP data. This is done in Figure 26 where the currents are shown at individual shotpoints, and Figure 25 where the currents (at each shot) are interpolated to the vessel location and then averaged over the available data (roughly one hours worth of inferred currents at each location - except at the start and end of the data). Noting that the ADCP data were used in the inverse scheme, there is a general consistency between the inferred $w_{y}$ and the ADCP data. Towards the end of the data set (left of the figures) the inferred $y$ currents seem consistently lower than ADCP currents, this is when the three DCUs are steering; and we have previously noted problems. The estimated $w_{x}$ seems to be generally lower than the ADCP currents throughout the experiment. This could be evidence of the fact that the inferred currents are at a later time than the ADCP currents, or it could be evidence of over smoothing.

Figure 24 shows the mean inferred currents, at a grid of points that the streamer array passes through, rather than just the vessel locations. From this figure we can see that there is variation in the currents across the streamers, and that the smoothness assumptions have not resulted in identical currents being inferred along each streamer.

Another check on the self consistency of the method is the estimated tension. Figure 27 shows that the inferred tension at the head of Streamer 11 closely follows the data and remain 

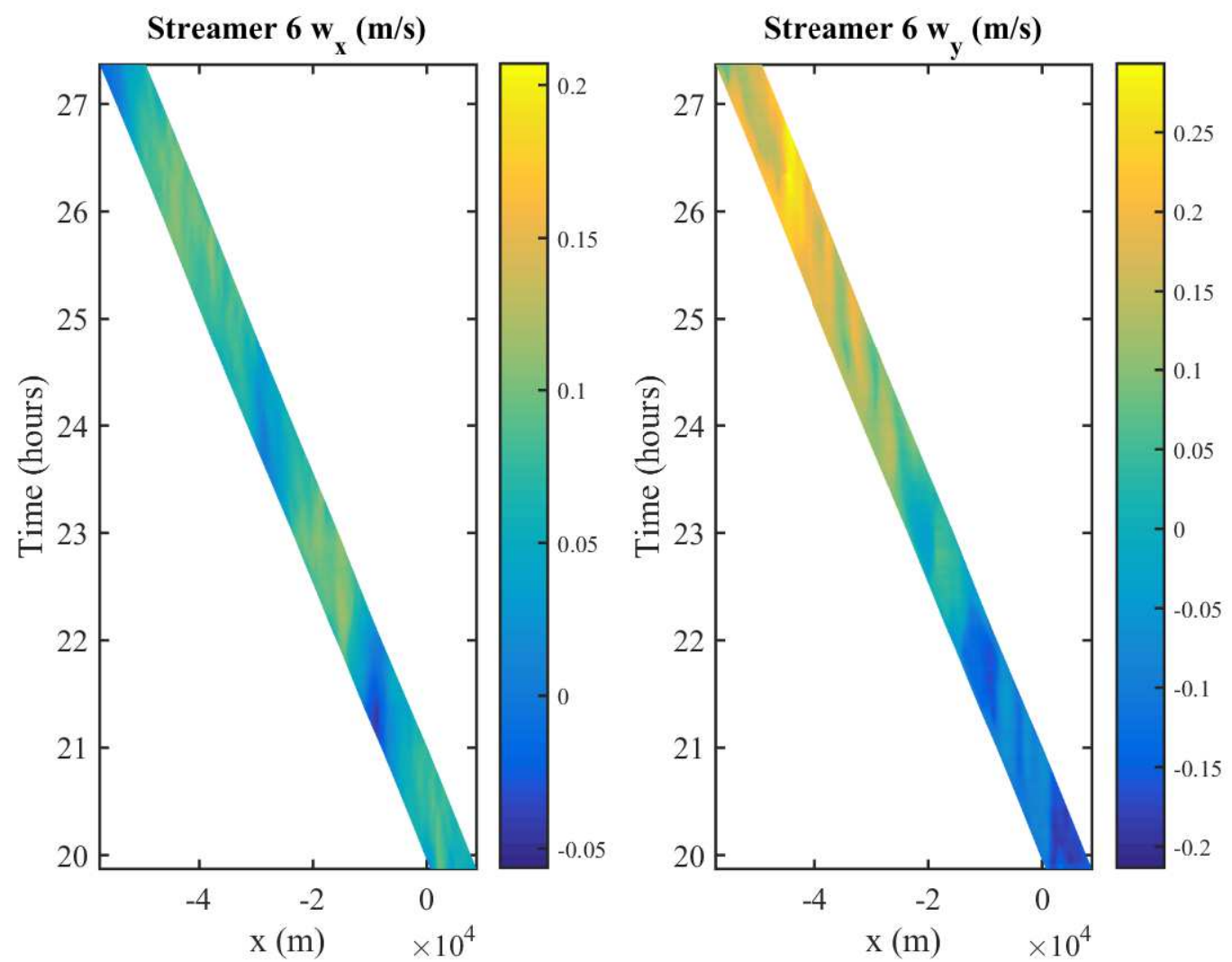

Figure 23. The inferred $x$ and $y$ currents at every time step along Streamer 6 , plotted against the $x$ location they were inferred at. Note that the vessel is traveling in the negative $x$ direction.

realistic at the tail end (for some choices of weights the tension can become negative near the tail end indicating that something is wrong). Looking at the estimated angle along a streamer in Figure 28 does not provide a self-consistency check, but the jumps in the angle are clearly visible when there is lateral steering which is as we would hope. 


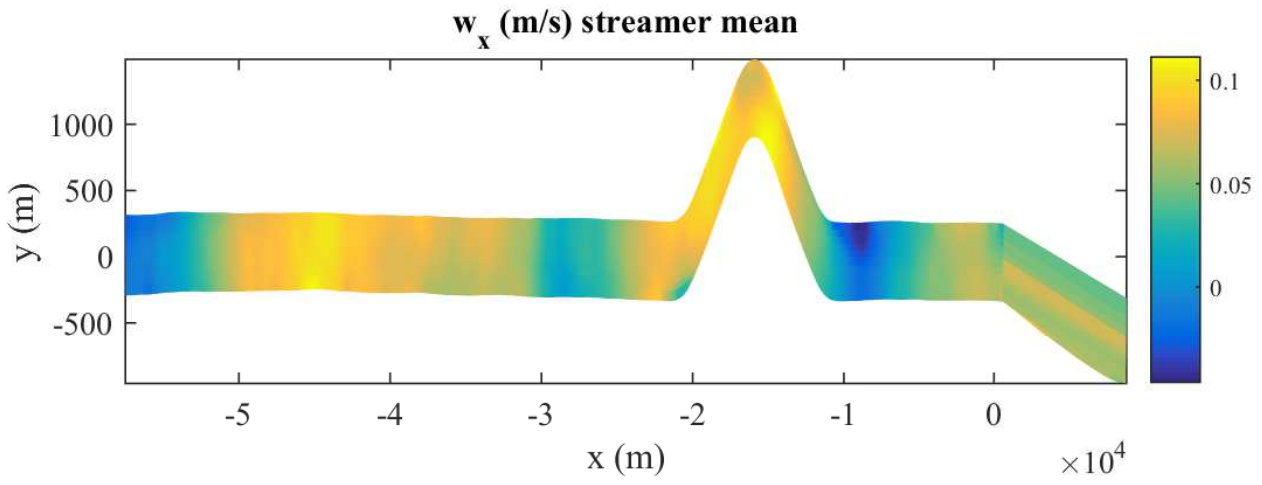

$w_{y}(m / s)$ streamer mean

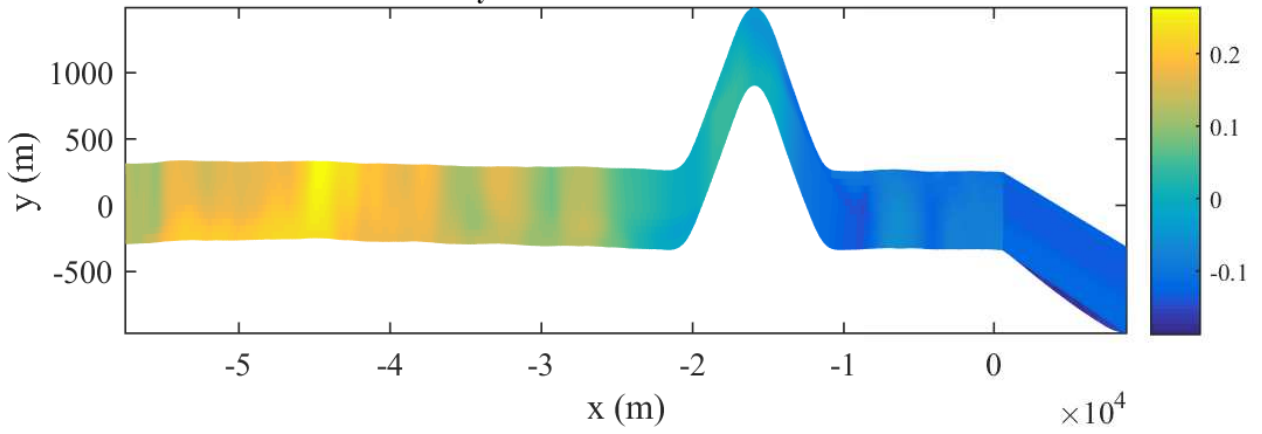

FiguRE 24. The mean inferred currents over the time that the streamer array covers that location. 

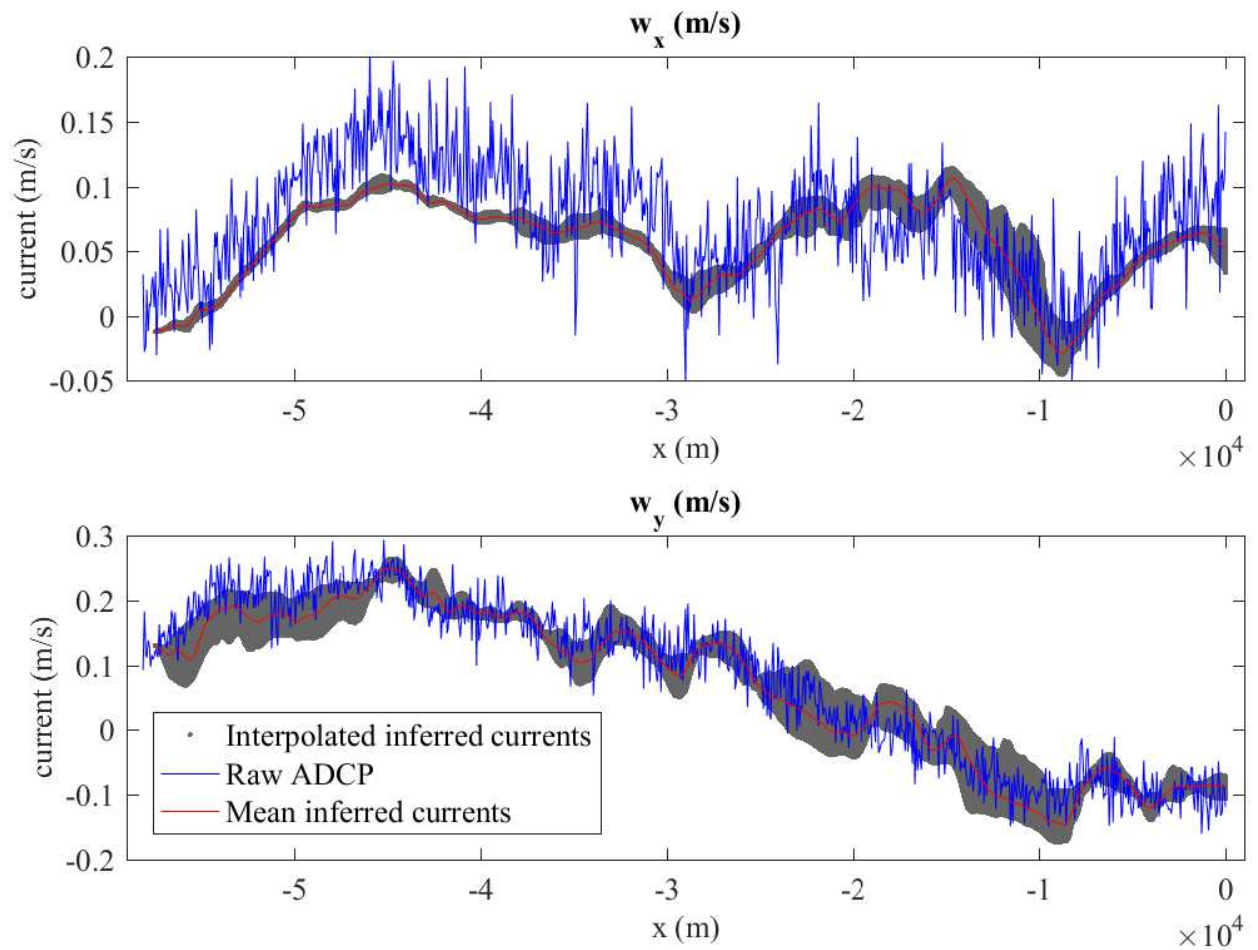

Figure 25. The mean inferred currents at the location the ADCP was recorded. Each light grey dot is the inferred current at a shot; the mean of these is given by the red line. 

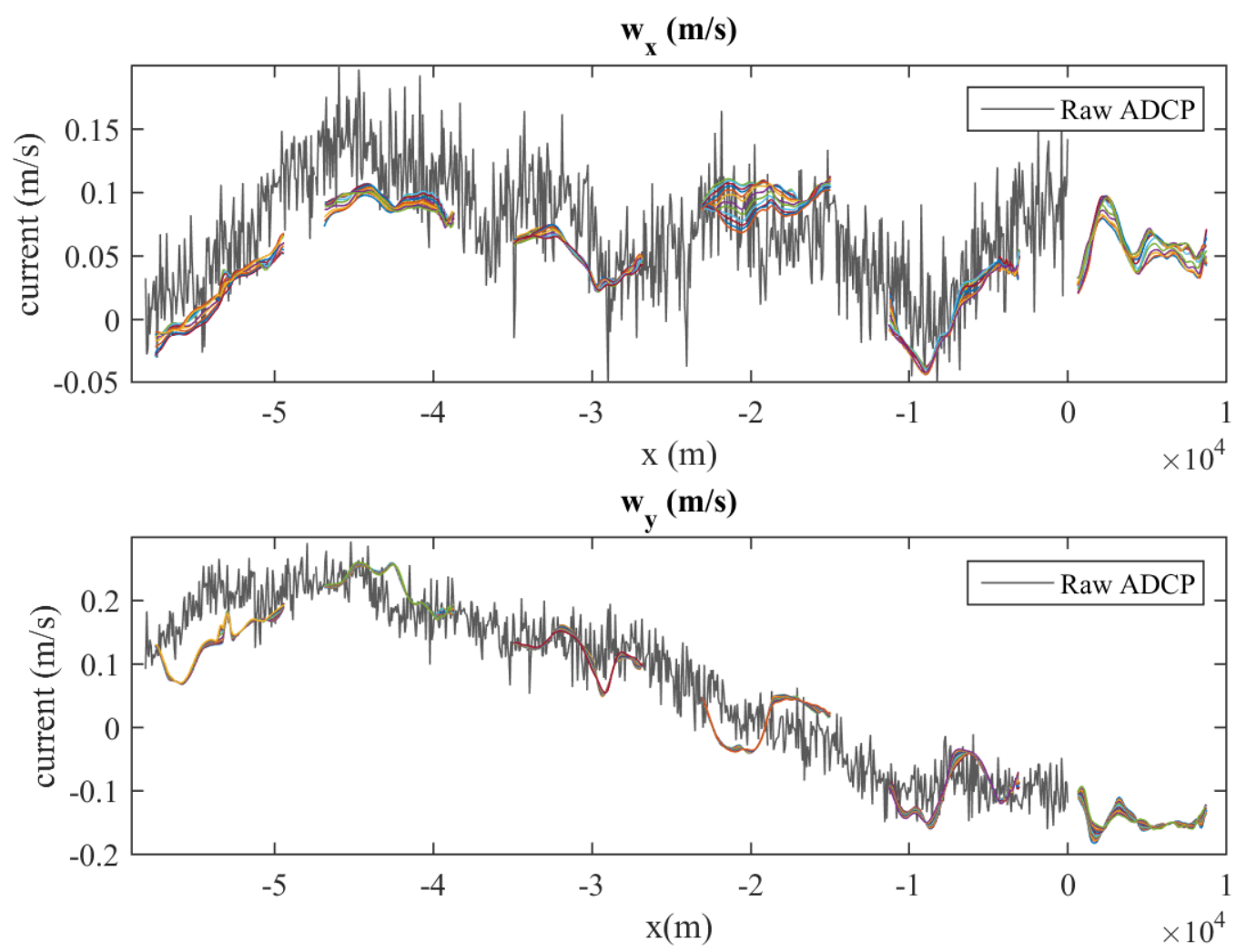

FiguRE 26. The inferred currents at different shotpoints (the array is traveling from right to left) compared with the currents recorded by the ADCP on the vessel. The inferred currents along each streamer have been plotted in different colours so that they can be distinguished. 


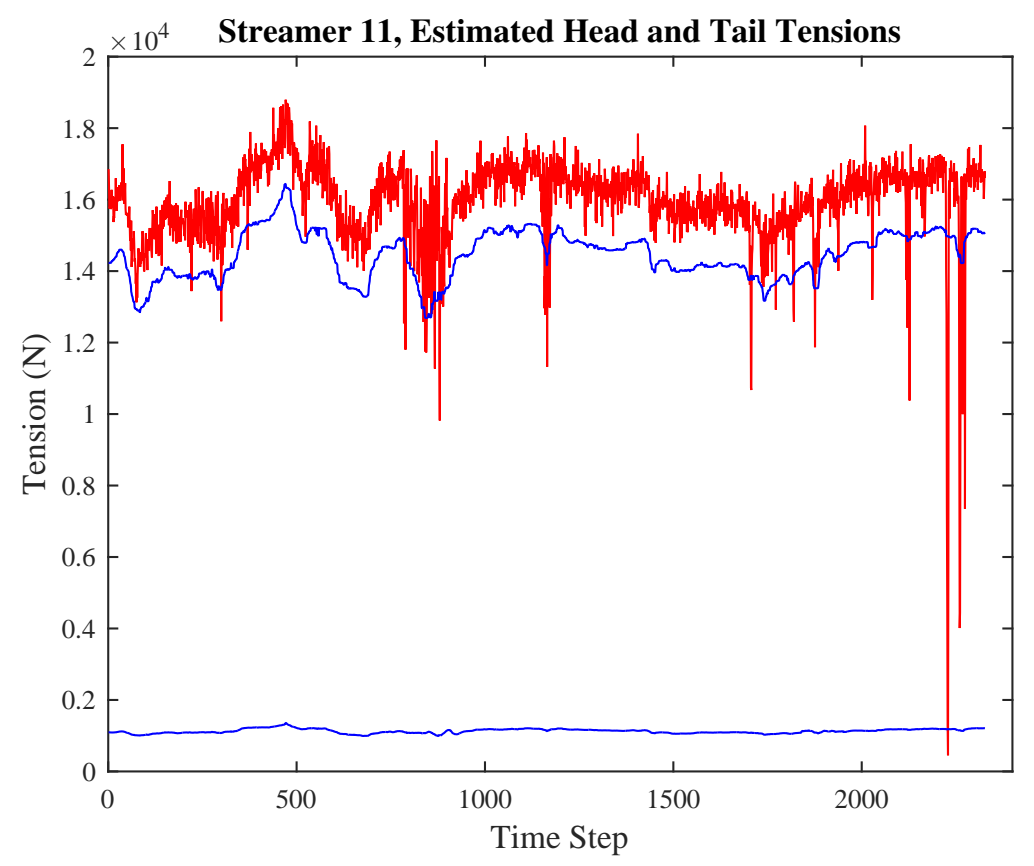

Figure 27. The inferred tension at the head and tail of Streamer 11 (top and bottom blue lines respectively), compared with the recorded tension (red). Note that, on this streamer, there is a miniwing and a front float between the head of the streamer and where the tension is recorded.

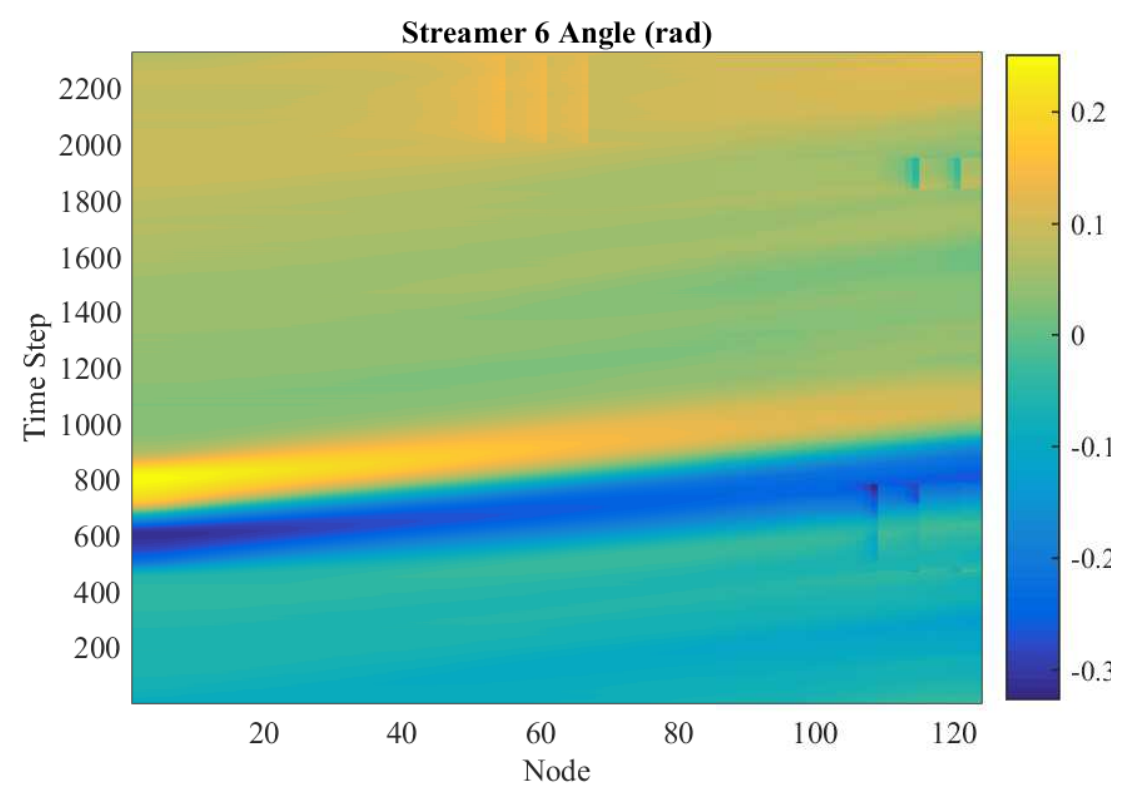

Figure 28. The inferred local angle inferred along Streamer 6. Note the jumps in the angle, that occur at the tail of the streamer around shots 600 and 1800, and near the centre of the streamer around shot 2000, that correspond to DCUs providing lateral steering. 

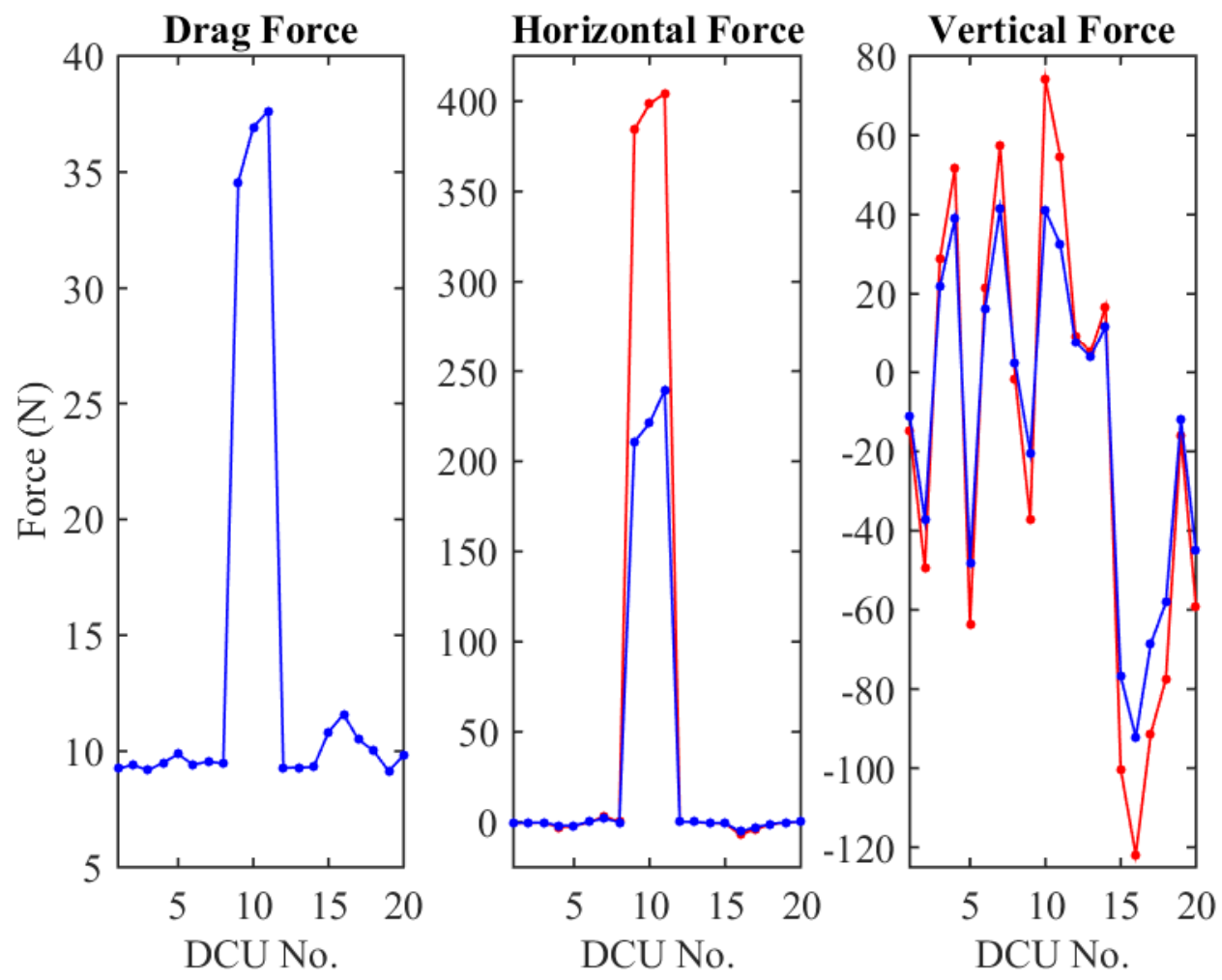

FIGURE 29. The inferred drag, horizontal force and vertical force (blue) for each DCU along Streamer 6 at a given shot, compared with the recorded force (red). The 'recorded force' is greater because the formula to calculate the force assumes a speed through the water of $2.57 \mathrm{~m} / \mathrm{s}$ ( 5 knots), the true speed is less than this. There also may be errors in the inferred currents which will affect the calculated force. 


\section{Discussion}

This paper demonstrates that it is possible to infer plausible ocean currents from the shapes of streamer arrays when there is no lateral steering. Estimating the crossline currents only requires a reasonable tension estimate and a sufficient smoothness weighting. The inline currents were estimated by increasing the divergence-free weight until coherent structures were detected in time. Using this method enables one to determine the minimum necessary divergence-free weight to estimate inline currents for a given smoothness weight (Figures 8 to 12 show that, as the smoothness weight is increased the greater the divergence-free weighting required to observe realistic inline currents). If the divergence-free weight is increased beyond this threshold, then the inline and crossline currents are slightly affected; hence, there remains a degree of uncertainty in the currents. Clearly, if a small error in the currents is acceptable, one could choose an arbitrarily large divergence-free weight, and, so just assume that the divergence-free assumption must be satisfied exactly (i.e. that there is no vertical motion detectable) and, so, the problem is reduced to just choosing the smoothness weight, which could be done by minimising equation (68). At the time of writing, choosing the weights is somewhat subjective (based on assumptions about the errors in the input variables and how smooth the currents should be in space and time), so, an important question is whether the choice of weights can be made automatic? A possible starting point might be found by treating the various weights as nuisance parameters ${ }^{6}$ (Golub and Pereyra 2003, Aravkin and Leeuwen 2012).

The greatest problem to inferring the currents is when there is significant steering. This is caused by errors estimating the velocity of the streamer and, possibly, errors in the position data leading to a reduced curvature. The problem is partly mitigated by assuming that the currents are smooth in time and that the velocity data have errors. However there remain problems in inferring the normal currents. To balance the normal force equation, the relative normal velocity of the streamer to the current is altered which affects the estimated DCU forces. If the position data show too small a change in angle, then the DCU force must be reduced from the actual force, leading to an error in the current. Similarly, if the velocity of the streamer is wrong then the inferred normal current will also be wrong so as to maintain the correct relative velocity. Therefore, if the currents are to be inferred using the methodology described, the velocity of the streamer needs to be reliably calculated to include jumps when the DCUs are turned on. This might be achieved by using a non-smooth form of regularization such as total variation regularisation when processing the velocities. Doing this would enable the smoothness in time criteria to be neglected from the cost function enabling it be used, solely, as a criteria for judging the plausibility of any inferred currents. More generally, knowing the velocities more accurately will mean that their residual weighting can be increased in the cost function and so signal is less likely to be lost from the currents and attributed to velocity errors.

This paper, whilst showing that it is possible to infer current-like structures from streamer position data, has not provided evidence that the inferred structures are indeed currents. The only measure of the currents available was the ADCP data that was used in the inversion

\footnotetext{
${ }^{6}$ Suggested by James Rickett, (Schlumberger Gould Research).
} 
process, and, so, all that has been shown is that the method is self-consistent. Therefore this analysis should be repeated on some streamer data that have independent measurements of the currents. This could be work boats with ADCPs placed at the tail and sides of the array, wave-gliders with current meters, drifters, or coastal radar ${ }^{7}$.

When repeating this work it would be better to work with the raw range and GPS data to infer the currents, or the output positions of the acoustic network, rather than using the calculated receiver positions and trying to undo the effects of the interpolation and processing. If the inferred currents are consistent with the independent observations, this suggests that the recalculated position data (whilst imitating the acoustic network) for the streamers may be more accurate than the original position data, and, so, leads to the question: do the new positions makes a difference to the seismic image or not?

If the new position data affects the seismic image then this may motivate inferring the currents as an off-line process rather than a real-time process (motivated by benefits to steering the streamers). This then opens up alternative methods for inferring the currents, along with new position data. Rather than solving the currents at each shotpoint the currents and positions could be inferred over a time window. This will greatly increase the computational cost (it may be possible with an adjoint method) but it should be possible to better handle the velocity jumps at DCUs because the velocity of the streamer would be calculated as part of the inversion and not be required as an input. Another benefit might be to treat the drag coefficients as unknowns (that are fixed in time) along each section, to reflect the different amounts of barnacles on each section. This could possibly be constrained slightly using the logs of when each streamer section was last cleaned. At present, there is some uncertainty in the inferred currents due to uncertainties in the drag coefficients, this probably forces us to search for currents that are smoother than would otherwise be necessary. This could then lead to more accurate currents and a diagnostic tool for determining which sections of streamer require cleaning next ${ }^{8}$. If both the off-line and real time inverse procedures can be made to work then they could be used in tandem: the inferred currents from the real time process could be used as an intial guess for the off-line inversion, and the estimated drag coefficients from the off-line process can then be used the next time the real time process is run.

\section{ACKNOWLEDGMENTS}

The author thanks Robert Laws for help and guidance throughout this project, Ken Welker for describing how the node positions are calculated, Nicolas Barral for discussing reasons why the drag on different streamers may be different, the seismic vessel Western Neptune for collecting the data and the many others at WesternGeco who helped with my queries. Finally the author thanks NERC (grant no. NE/L008246/1) and Schlumberger for funding this research.

\footnotetext{
${ }^{7}$ Suggested by Emily Shuckburgh (Britsh Antarctic Survey).

${ }^{8}$ Suggested by Robert Laws (Schlumberger Gould Research).
} 


\section{REFERENCES}

Aravkin A. and Van Leeuwen T. 2012. Estimating nuisance parameters in inverse problems. Inverse Problems 28(11).

Chartrand R. 2011. Numerical Differentiation of Noisy, Nonsmooth Data. ISRN Applied Mathematics 2011.

Golub G. and Pereyra V. 2003. Separable nonlinear least squares: The variable projection method and its applications. Inverse Problems 19(2), R1-R26.

Grant T.J., Laws R.M. and Shuckburgh E.F. 2014. Estimating ocean currents from the shapes of laterally steered streamer arrays. First Break 32, 57-66.

Hanke-Bourgeois M. and Scherzer O. 2001. Inverse problems light: Numerical differentiation. American Mathematical Monthly 108(6), 512-521.

Kragh E. and Christie P. 2002. Seismic repeatability, normalized rms, and predictability. The Leading Edge 7, 640-647.

Lighthill J. 1986. An informal introduction to theoretical fluid mechanics. The institute of mathematics and its applications monograph series. Oxford University Press.

MathWorks 2014. lsqnonlin. http://uk.mathworks.com/help/optim/ug/lsqnonlin.html. Accessed: $27 / 11 / 2014$.

Polydorides N. and Storteig E. 2011. Ocean current inference using towed cable hydrodynamics. Journal of Waterway, Port, Coastal and Ocean Engineering 138(1), 2-8.

Polydorides N., Storteig E. and Lionheart W. 2008. Forward and inverse problems in towed cable hydrodynamics. Ocean Engineering 35(14-15), 1429-1438.

Polydorides N., Storteig E. and Lionheart W. 2009. Ocean current prediction in towed cable hydrodynamics under dynamic steering. Inverse Problems in Science and Engineering 17(5), 627-645.

Thomas L., Tandon A. and Mahadevan A. 2008. Submesoscale processes and dynamics. In: Ocean Modeling in an Eddying Regime (eds. M.W. Hecht and H. Hasumi). American Geophysical Union.

Vallis G.K. 2006. Atmospheric and Oceanic Fluid Dynamics. Cambridge University Press.

Wunsch C. 2006. Discrete Inverse and State Estimation Problems with Geophysical Fluid Applications. Cambridge University Press.

E-mail address: timothy_grant@hotmail.co.uk

Schlumberger Gould Research, High Cross, Madingley Road, Cambridge, CB3 0EL, UK.

British Antarctic Survey, High Cross, Madingley Road, Cambridge, CB3 0Et, UK. 